\title{
WestVirginiaUniversity
}

THE RESEARCH REPOSITORY @ WVU

Graduate Theses, Dissertations, and Problem Reports

2002

\section{Perceived efficacy and utilization of the OralCDx brush biopsy}

Courtney Niccole Huffman

West Virginia University

Follow this and additional works at: https://researchrepository.wvu.edu/etd

\section{Recommended Citation}

Huffman, Courtney Niccole, "Perceived efficacy and utilization of the OralCDx brush biopsy" (2002). Graduate Theses, Dissertations, and Problem Reports. 1472.

https://researchrepository.wvu.edu/etd/1472

This Thesis is protected by copyright and/or related rights. It has been brought to you by the The Research Repository @ WVU with permission from the rights-holder(s). You are free to use this Thesis in any way that is permitted by the copyright and related rights legislation that applies to your use. For other uses you must obtain permission from the rights-holder(s) directly, unless additional rights are indicated by a Creative Commons license in the record and/ or on the work itself. This Thesis has been accepted for inclusion in WVU Graduate Theses, Dissertations, and Problem Reports collection by an authorized administrator of The Research Repository @ WVU. For more information, please contact researchrepository@mail.wvu.edu. 


\title{
PERCEIVED EFFICACY AND UTILIZATION OF THE ORALCDX BRUSH BIOPSY
}

\author{
Courtney N. Huffman BSDH \\ Thesis submitted to the \\ School of Dentistry \\ at West Virginia University \\ in partial fulfillment of the requirements \\ for the degree of \\ Master of Science \\ In \\ Dental Hygiene
}

Christina B. DeBiase, Ed. D., Chair

Jerry E. Bouquot MDS

Richard L. Meckstroth DDS

Carol A. Spear MSDH

Department of Dental Hygiene

Morgantown, West Virginia

2002 


\section{ABSTRACT}

Perceived Efficacy and Utilization of the OralCDx Brush Biopsy

\section{Courtney Huffman}

The OralCDx is a preliminary screening tool developed to identify suspicious lesions. Despite large numbers of persons diagnosed with oral cancers annually, minimal research has been conducted on the efficacy and utilization rates of OralCDx. The purpose of this study is to determine perceived efficacy and utilization rates of OralCDx nationwide. A 16-item survey was used to survey a random sample of 100 dentists and a 6-item survey was used to survey a random sample of 400 patients. Data analyses were conducted using frequencies, cumulative frequencies, percents, analysis of variance, and tests of chi-square using the JMP program. Preliminary results revealed that $54 \%$ of the dentists had high/extremely high confidence in the accuracy of the results. Preliminary results revealed $81 \%$ of the patients reported no pain involvement with OralCDx. It can be concluded form this study that OralCDx is accepted by both dentists and patients as a non-invasive screening tool. 


\section{DEDICATION}

To my husband Aaron, my best friend, for his unconditional love and support, and for the constant joy he brings to my life. He is truly a gift from God.

To my family for their continual prayers, love, support, and patience with my educational endeavors.

To Christina DeBiase, my mentor, for her wisdom, guidance, and her love of students, education, and dental hygiene. 


\section{ACKNOWLEDGEMENTS}

The author would like to express her deepest gratitude to her thesis committee: Dr. Christina B. DeBiase, Dr. Richard L. Meckstroth, Dr. Jerry E. Bouquot, and Mrs. Carol A. Spear for their patience, expert advice, support, and warmth throughout all stages of this study. I could not imagine accomplishing my goals without each of them.

The author is especially grateful to Medical Director of OralScan Laboratories Inc., Dr. Drore Eisen, for his willingness to help through all stages of this study despite his tireless schedule.

The author is sincerely grateful for the intellectual mind of Dr. Endogen Gunel for his kindness and his incredible knowledge and help with statistics.

The author would like to express thanks to Loreen Hurley and Mary Monahan for the tireless phone calls and requests I made throughout this study. You are very much appreciated. A special thanks to my brilliant husband Aaron, for his constant support and assistance with all of the statistical graphs.

Finally, the author would like to thank God who gave me the abilities to accomplish my goals. And to my family, who I have not seen a lot of throughout my educational travels, for their love and continual support through all my educational dreams.

God bless you all! 


\section{TABLE OF CONTENTS}

PERCEIVED EFFICACY AND UTILIZATION OF THE ORALCDx BRUSH BIOPSY ............

ABSTRACT

DEDICATION

ACKNOWLEDGEMENTS ....................................................................... iv

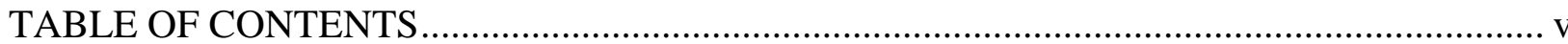

LIST OF FIGURES

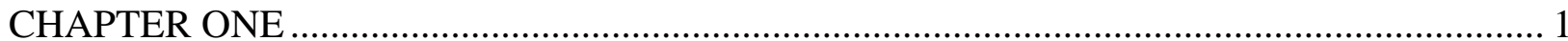

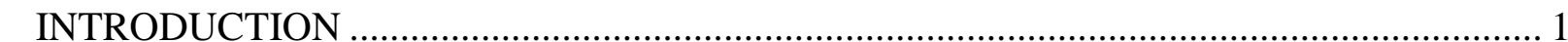

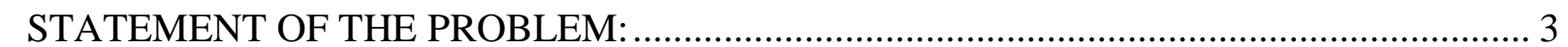

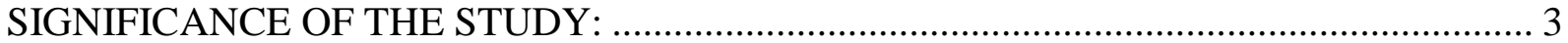

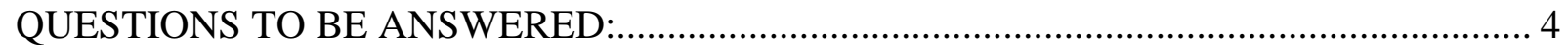

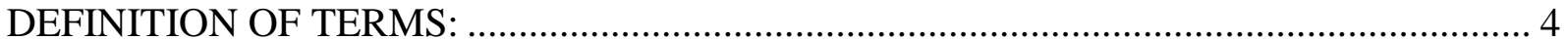

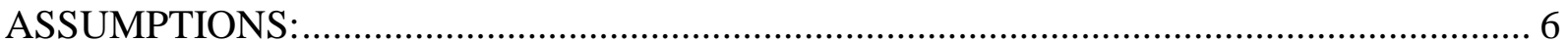

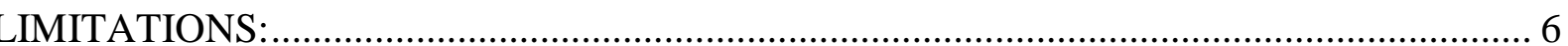

DELIMITATIONS:

CHAPTER TWO.

LITERATURE REVIEW

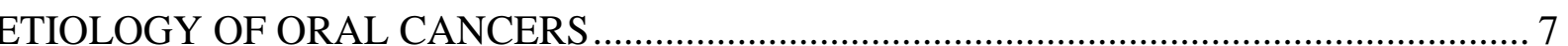

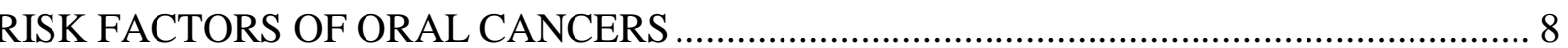

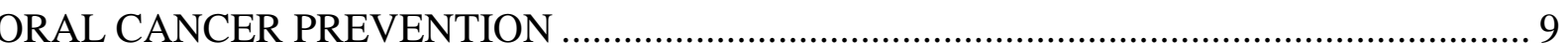

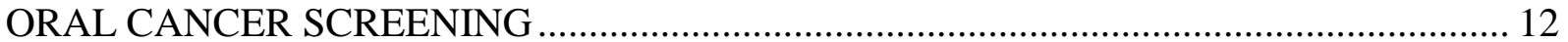

ORALCDx . ...

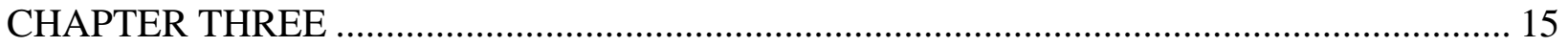

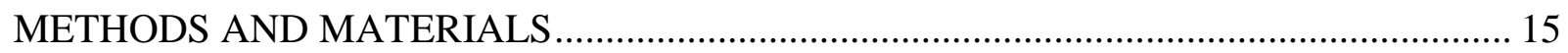

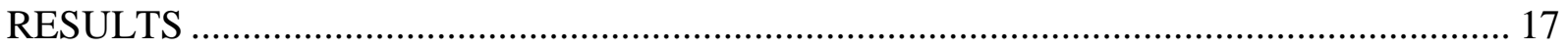

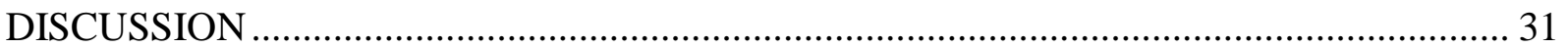

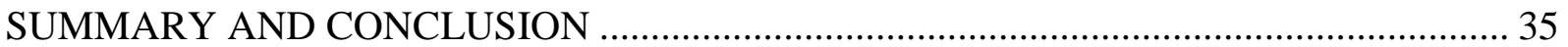

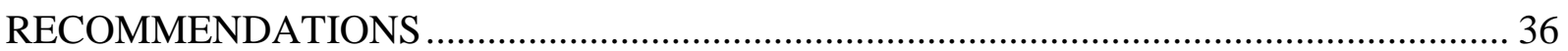

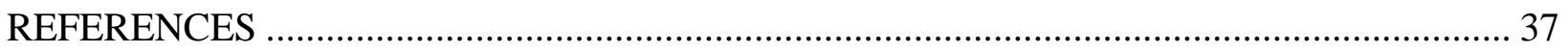

APPENDIX A ……................................................................................................ 39

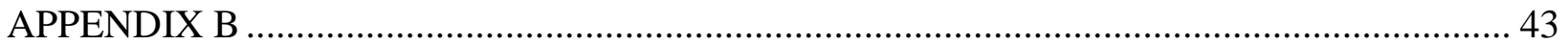

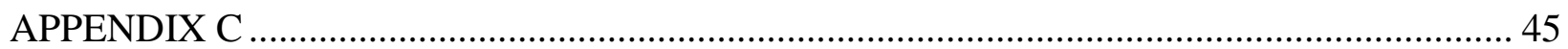

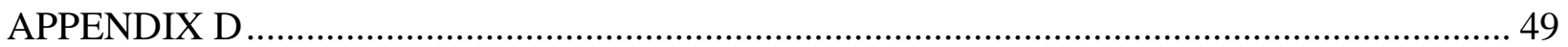

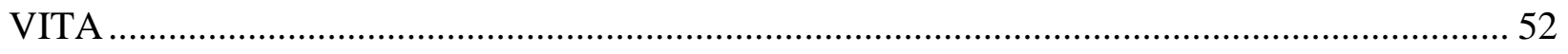




\section{LIST OF FIGURES}

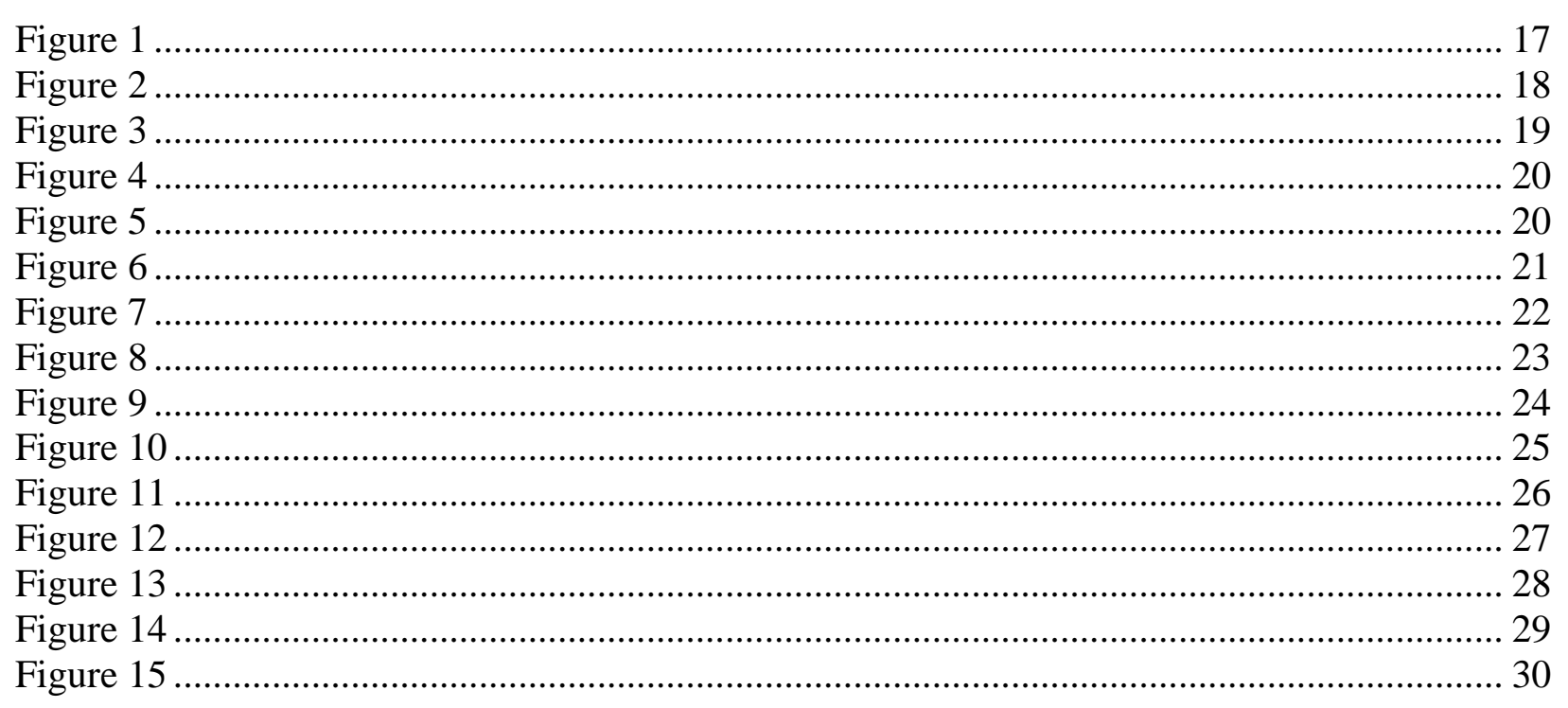




\section{CHAPTER ONE}

\section{INTRODUCTION}

Oral cancer is a disorder involving malignant tissue growth in the oral cavity. Different cancers can develop from individual types of cells. Depending on the types of cells involved and the extent of neoplasmic growth, oral lesions are diagnosed as benign or malignant. Diagnosis is important because it influences patient treatment options and outlook for recovery. Some oral cancers display symptoms that cause patients to seek medical attention; however, most oral cancers do not cause symptoms until they have reached an advanced stage. Treatment varies widely depending on many variables that include location, type, and stage of the cancer at the time of diagnosis. Some oral cancers require localized surgical excision, while others may require extensive surgical removal that results in severe physical deformities with subsequent psychological consequences ${ }^{1}$.

Although the average at age diagnosis is 60 , ninety five percent of oral cancer occurs primarily in adult males over 40 years of age ${ }^{2}$. Over 30,000 cases of oral cancers are diagnosed annually and result in over 8,000 deaths in the United States each year ${ }^{2}$. Although early diagnosis has the best long-term prognosis, many oral cancers remain undiagnosed or are not diagnosed until a late stage. Despite efforts to reduce oral cancer and high-risk behaviors, the stage at diagnosis has remained unchanged in the past 50 years ${ }^{2}$. Over $25 \%$ of oral cancer victims have not used tobacco and have had no other known high-risk factors ${ }^{3}$.

The exact etiology of oral cancer is unknown. Smoking tobacco, smokeless tobacco use, and heavy alcohol consumption are documented in the literature as high-risk factors associated with most cases of oral cancers. These factors are frequently associated with poor dental and oral hygiene. 
Regular dental exams are vital for early detection of oral cancer and especially oral precancers. Two well-known lesions identified as precancerous lesions in the oral cavity are leukoplakia and erythroplakia. Leukoplakias are white, slightly raised areas that are not likely to be malignant. At the initial diagnosis, approximately $5 \%$ of leukoplakias are cancerous. If leukoplakias are improperly treated, they have an increased risk of progressing into cancer within ten years. Erythroplakias are slightly raised reddish areas that cannot be scraped off. Fifty-one percent of erythroplakias are diagnosed as cancer at the initial biopsy ${ }^{4}$. Incisional biopsy is required to determine final diagnosis and to determine if oral lesions are diagnosed as benign or malignant.

The large numbers of persons diagnosed annually with oral cancers may be identified earlier by a new screening technique called the OralCDx brush biopsy. However, there is limited information available concerning the efficacy and utilization of the OralCDx brush biopsy in screening suspicious oral lesions at an early stage. Since oral cancer has the best prognosis when diagnosed early, it is vital to determine the utilization and efficacy of the OralCDx brush biopsy by dental professionals. It has been surmised that many dental professionals monitor suspicious lesions or directly refer patients to a specialist to perform incisional biopsies. Although the OralCDx brush biopsy is being researched as a less invasive means of screening for oral cancer, it does not eliminate the need for the more invasive incisional biopsy for final diagnosis.

The purpose of this research was to determine nationally, the utilization and perceived efficacy of the OralCDx brush biopsy by dental professionals who have performed the biopsy and patients who have received the biopsy. Perceived efficacy is defined as the dentists' identification of lesions for the OralCDx brush biopsy, performance of the biopsy, confidence with the results, and attitudes related with these experiences. 


\section{STATEMENT OF THE PROBLEM:}

Limited research has been performed concerning efficacy of the OralCDx brush biopsy, and furthermore no research has been published on professional and patient acceptance.

\section{SIGNIFICANCE OF THE STUDY:}

It is documented in the literature that persons having an early diagnosis and intervention of pre-cancerous and cancerous oral lesions at an early stage have a better overall long-term prognosis as compared to persons having undiagnosed or advanced stage diagnosis of oral lesions. The lack of early detection may be attributed to lack of annual oral cancer screening examinations, minimal patient understanding of the early signs and symptoms of oral cancers, and popularity of high-risk behaviors and habits, including environmental factors. Persons having an early diagnosis of oral cancer have an $80 \%$ chance of five-year survival ${ }^{1}$. Late stage detection of oral lesions can lead to dramatic physical and psychosocial post surgical consequences ${ }^{1}$.

In order to avoid this problem, numerous methods have been suggested. The OralCDx brush biopsy is the newest technique. Dentists and dental hygienists may be more apt to use the OralCDx brush biopsy due to minimal patient discomfort. No research has been published concerning the utilization and efficacy of the OralCDx brush biopsy technique. Because the OralCDx brush biopsy is designed for early screening and detection, it is vital to determine the efficacy and utilization rate of this screening tool in order to promote health and longevity.

The purpose of this study is to determine the professional perceptions of efficacy and professional utilization rates of the OralCDx brush biopsy and to determine patient perceptions involved in the procedure. Perceptions, utilization, and perceived efficacy will be evaluated by 
dental professionals who have performed the OralCDx brush biopsy and by patients who have received the biopsy.

The results will provide information about the usefulness and necessity of using the OralCDx brush biopsy as a screening tool in order to detect oral cancers at an early stage. The results will help determine the perception of professionals as to whether or not there is an association between using the OralCDx brush biopsy and accurate early detection of suspicious oral lesions. If there is an association, then education about utilizing the OralCDx brush biopsy by dental professionals will help to further emphasize the importance of early oral cancer detection in a less invasive manner.

\section{QUESTIONS TO BE ANSWERED:}

1. Is there an association between the number of dentists who have high/extremely high confidence in the OralCDx brush biopsy and the number of biopsies they have performed?

2. Is there an association between dental offices that have dental hygienists performing OralCDx brush biopsies and the location of the dental office?

3. Is there an association between dentists who feel that all suspicious lesions should have OralCDx brush biopsies and the number of biopsies that have reported positive/abnormal results?

4. Is there an association between the number of years a dentist has practiced dentistry and his/her confidence level in the accuracy of the results of the OralCDx brush biopsy?

5. Is there an association between the number of positive/abnormal results and where the information for the OralCDx brush biopsy was obtained?

\section{DEFINITION OF TERMS:}

Biopsy/Incisional Biopsy- removal of tissue for microscopic diagnosis

Screening- preliminary procedure to determine if further treatment is needed

Non-invasive- a procedure that does not require the skin to be broken 
Exfoliative Cytology- scraping cells on the surface of tissue for microscopic diagnostic purposes.

Suspicious Oral Lesion- any abnormal epithelial lesion in the mouth that would not be selected for immediate histologic exam.

Benign- localized tumor having well differentiated cells that do not invade surrounding tissues or metastasize.

Malignant- anaplastic, invasive that tends to spread and end in death.

Sensitivity- quality of the ability to detect

Specificity- quality of being distinctive

Positive- showing disease

Negative- showing absence of disease

False-Negative- showing no detection of disease when disease is present

False-Positive- showing the presence of disease when no disease is present

International TNM System of Classification of Oral Carcinomas: ${ }^{5}$

T, Size of tumor

$\mathrm{T}_{1 \mathrm{~s}}$, Carcinoma in situ

$\mathrm{T}_{1}$, Tumor $<2 \mathrm{~cm}$ in size

$\mathrm{T}_{2}$, Tumor $>2 \mathrm{~cm}$ to $<4 \mathrm{~cm}$ in size

$\mathrm{T}_{3}$, Tumor $>4 \mathrm{~cm}$ in size

$\mathrm{T}_{4}$, Massive tumor with deep invasion into bone, muscle, skin, etc.

$\mathrm{N}$, Regional lymph node involvement

$\mathrm{N}_{0}$, No palpable nodes

$\mathrm{N}_{1}$, Single, homolateral palpable node $<3$ in diameter

$\mathrm{N}_{2}$, Single, homolateral palpable node, 3 to $6 \mathrm{~cm}$, or multiple, homolateral nodes, none $>6 \mathrm{~cm}$

$\mathrm{N}_{3}$, Single or multiple, homolateral nodes, one $>6$ $\mathrm{cm}$, or Bilateral nodes, or contralateral nodes

M, Metastases

$\mathrm{M}_{0}$, No known metastasis

$\mathrm{M}_{1}$, Distant metastasis 


$$
\begin{array}{ll}
\text { I } & \mathrm{T}_{1}, \mathrm{~N}_{0}, \mathrm{M}_{0} \\
\text { II } & \mathrm{T}_{2}, \mathrm{~N}_{0}, \mathrm{M}_{0} \\
\text { III } & \mathrm{T}_{3}, \mathrm{~N}_{0}, \mathrm{M}_{0} \\
& \mathrm{~T}_{1}, \mathrm{~T}_{2}, \text { or } \mathrm{T}_{3}, \mathrm{~N}_{1}, \mathrm{M}_{0} \\
\text { IV } & \text { Any } \mathrm{T}, \mathrm{N}_{2}, \mathrm{~N}_{3}, \mathrm{M}_{0} \\
& \text { Any } \mathrm{T}, \text { any } \mathrm{N}, \mathrm{M}_{1}
\end{array}
$$

\section{ASSUMPTIONS:}

1. Dental professionals perform the OralCDx brush biopsy with correct technique and proper training.

2. Patients may not be aware of the significance of their oral condition and the availability of diagnostic techniques.

3. In compliance with the researcher's requests, the Medical Director of OralScan Laboratories Inc. randomly selected participants, sent surveys, received, reviewed, and tabulated results.

4. Patients want any screenings/biopsies performed to provide accurate results.

5. The OralCDx is a professionally accepted non-invasive screening tool.

\section{LIMITATIONS:}

1. The researcher did not have complete control over the dentist and patient selection and explanation of high-risk behaviors/habits, patient survey mailing, receiving, or tabulation of results.

2. No follow-up survey will be distributed to determine final diagnosis.

3. Participants included only those dentists already familiar with the procedure.

\section{DELIMITATIONS:}

1. Dentists selected for this study must have performed the OralCDx brush biopsy on a minimum of one patient.

2. Patients selected for this study must have received at least one OralCDx brush biopsy procedure on a suspicious lesion.

3. Participants in this study must be 18 years of age or older.

4. All respondents are geographically located in the United States. 


\section{CHAPTER TWO}

\section{LITERATURE REVIEW}

"Oral cancer is a common disorder that is associated with significant morbidity and mortality. Because it often goes undiagnosed, treatment options are limited and prognosis is affected"6 . Approximately 30,000 cases of oral cancers are diagnosed annually ${ }^{7}$. Despite technological advances, "at stage diagnosis has not changed significantly in the past decade". Early detection and diagnosis includes patient education, annual oral cancer screening examinations by dental professionals, and eliminating patients' high-risk behaviors and habits.

\section{ETIOLOGY OF ORAL CANCERS}

In 1998, the Center for Disease Control (CDC) reported an estimated 30,300 diagnosed cases of oral cancers. "Approximately 8,000 deaths (5,200 males and 2,800 females) are expected each year. Ninety-five percent of oral cancer cases occur among persons aged over 40 years, and the average age at diagnosis is 60 years" ${ }^{\prime 2}$. High-risk behaviors and habits put patients at risk for developing oral cancers. High risk behaviors and habits include smoking cigarettes, pipes, or cigars, consuming large amounts of alcohol, use of smokeless tobacco, and environmental and lifestyle habits that include exposure to sunlight and diet. Tobacco smoking and heavy alcohol consumption (over 30 drinks per week) are primary risk factors for developing oral cancers and account for $75 \%$ of oral cancers in the United States ${ }^{2}$. Oral cancers arising from primary high-risk behaviors can be prevented through tobacco cessation and decreasing alcohol consumption. The remaining $25 \%$ of oral cancers are of unknown origin ${ }^{3}$. The literature states that $36 \%$ of persons have localized disease, $43 \%$ of persons have regional disease, and $9 \%$ of persons have distant disease at the time of diagnosis ${ }^{2}$. Early diagnosis and detection of oral cancers is vital for long-term prognosis and longevity. "The five-year survival rate for persons 
having oral cancers is $81 \%$ for those with localized disease, $42 \%$ for patients with regional disease, and $17 \%$ for those with distant metastases". Over ninety percent of oral cancers are diagnosed as squamous cell carcinomas ${ }^{2}$. Boyle, Macfarlane, and Scully state that physicians, dentists, and pharmacists, should have the knowledge of early detection of oral lesions and the importance of seeking advice from a specialist. Education and behavior modifications are still the primary preventions for oral cancers. "When a person stops smoking, the risk of oral cancer drops to that of a lifelong non-smoker after five to ten years after smoking cessation" ${ }^{\prime 8}$

\section{RISK FACTORS OF ORAL CANCERS}

"Smoking, a once socially accepted behavior, is the leading cause of preventable death and disability in the United States"'. Risk factors for cancers of the mouth include tobacco and alcohol use, sun exposure, increase in age, and previous history of cancer. Smoking alone accounts for $75 \%$ of all oral cancers in the United States ${ }^{2}$. A 1994 study was performed to evaluate the relation of smoking and alcohol consumption to the development of secondary tumors in high-risk groups. Patients having high-risk behaviors with the occurrence of secondary tumors were selected over a one-year period. Eighty patients were selected who displayed secondary tumors and the control group involved 189 patients who did not display secondary tumors. Results indicated that tobacco smoking and alcohol consumption contributed to the risk of secondary tumors. Smoking had more pronounced effects than alcohol consumption. Oral and pharyngeal cancers occurred in patients having the largest amounts of alcohol and smoking intakes ${ }^{10}$. The combination of smoking with alcohol usage has strong synergistic actions that increase oral cancer risk. Mucci and Brooks telephone surveyed 2119 Massachusetts adults aged 35 and older with a long smoking history in order to identify if these persons were less likely to visit the dentist annually. Compared to persons without either risk 
factor, those who were at higher risk from both long-term smoking and low fruit and vegetable consumption were even less likely to visit the dentist compared to persons without either risk factor. Also, the number of persons seeking annual exams decreased while their smoking duration and amount smoked per day increased ${ }^{11}$. Bouquot and Meckstroth demonstrated that although West Virginia had the highest per capita consumption of smokeless tobacco, it had less oral and pharyngeal cancers than the United States average. Results showed that average annual oral and pharyngeal cancer incidence in West Virginia males and females was 13.4 and 5.1, respectively, compared to the United States average of 15.4 and 5.7 (per 100,000 population) during $1950-1980^{12}$.

\section{ORAL CANCER PREVENTION}

It has been well documented in literature that oral cancer, if diagnosed at an early stage, has the best prognosis. Oral cancer can be identified as a lifestyle disease and, therefore, is primarily preventable. According to Diamond, et al. early detection and treatment of oral cancer is often successful, however, most cancers are detected at a late stage. As a result, late stage diagnosis leads to major physical and psychosocial morbidity, and poor prognosis. 'The past four years has driven to raise the profile of the prevention, and more specifically, the early detection of oral cancer ${ }^{1}$ Literature by Downie, et al. states that prevention of oral cancer and its impact on an individual have four focal points:

1. Reduce and avoid the risk of developing the illness

2. Focus on early diagnosis and effective treatment to restore health

3. Prevent complications associated with the disease and treatment

4. Prevent re-occurrence 
By targeting these four focal points focus needs to be centered around reducing oral cancer risk factors while simultaneously emphasizing advantages of early oral cancer detection and treatment. It must also indicate that diagnosis of cancer does not have to be a death sentence ${ }^{1}$. The importance of communicating to the population of prevention and early intervention is implacable. Johnson and Warnakalusuriya state that Health promotion campaigns for oral cancer have been primarily based on the broad population, yet research indicates that activities should target high-risk groups rather than the population as a whole. Client-centered activities are more sensitive to factors that influence individual readiness to change. Patients must be reached at a personal level in order to intervene with behaviors such as smoking cessation, reducing alcohol consumption, and utilizing dental services ${ }^{1}$. Although the United States government has established public awareness about the risks of using tobacco and alcohol, it cannot be guaranteed that public behavioral changes will follow. In 1997 the National Institute of Dental Research of the National Institute of Health and the American Dental Association held a conference concentrating on oral and pharyngeal cancer prevention, treatment, and research, from both public and private sectors. Participants at the conference developed recommendations concerning advocacy, collaboration, and coalition building; public education, professional education and practice; and data collection. The ten strategies selected for priority implementation included the following:

1. Establish a mechanism to implement and monitor the recommended strategies developed during the conference

2. Urge oral health professionals to become more actively involved in community health

3. Require instruction in preventing and controlling tobacco and alcohol use at all levels of training in dental, medical, nursing, and other related health-care disciplines 
4. Encourage Medicaid, Medicare traditional insurance plans, and managed-car entities to consider making oral cancer examinations an integral part of comprehensive physical and oral examinations

5. Designate federal funding for a national program of oral cancer prevention, early detection, and control

6. After assessing local needs, develop, implement, and evaluate statewide models to educate all relevant groups

7. Develop and conduct a national promotional campaign to raise public awareness of oral cancer and its link to tobacco use and heavy alcohol consumption

8. Develop health-care curricula that require competency in prevention, diagnosis, and multidisciplinary management of oral and pharyngeal cancer

9. Sponsor and promote continuing education for health-care professionals on the multidisciplinary management of all phases of oral cancer and its siquelae

10. Strengthen organizational approaches to reducing oral cancer by developing organized cooperative and collaborative arrangements, funding formal centers, and involving commercial firms

The strategies will be used by the Centers for Disease Control and Prevention (CDC) to develop programs to reduce the burden of oral and pharyngeal cancer in the United States ${ }^{2}$. A study of 251 fourth year dental students at three Texas dental schools were surveyed about their attitudes and perceptions of their knowledge and skills in areas related to oral cancers. A 16-item survey contained questions related to prevention, detection, diagnosis, and management of oral cancer and oral cancer therapy methods. A 79\% response rate was received. Over half of the students had observed a biopsy of oral tissue or read a report. However, only one-fourth had actually performed the procedure. Most respondents felt they were comfortable with their skills in counseling patients about oral cancer risk factors and most felt they were comfortable identifying a malignant lesion. Almost all respondents had been taught full head and neck examinations. Eighty-four percent were comfortable with their skills in performing these evaluations ${ }^{13}$. 
Many changes in the oral cavity occur in the retromolar area that is out of normal view for the patient.

\section{ORAL CANCER SCREENING}

It is well established that when oral cancer is diagnosed early, prognosis and treatment options are more positive. Education, annual examinations, and reduction of high-risk behaviors increase the ability of detecting oral cancer at an early stage. Two abnormal lesions identified as precancerous lesions in the oral cavity are leukoplakia and erythroplakia. Leukoplakias are white, slightly raised areas that are not likely to be malignant. Approximately $5 \%$ of leukoplakias are cancerous at initial diagnosis or, if not treated properly, have an increased risk of progressing into cancer within ten years. Erythroplakias are slightly raised reddish areas that cannot be scraped off. Fifty-one percent of erythroplakias are diagnosed as cancer at the initial biopsy $^{4}$. However, incisional biopsy is required to determine final diagnosis.

Oral exfoliative screening techniques are being utilized to determine cytology of suspicious oral lesions. It has been argued that only a small number of cells are evaluated in a cytologic smear, and therefore, it is not a valuable screening tool. The OralCDx brush biopsy was developed to identify lesions that warrant further diagnostic evaluation. A study involving 190 participants was conducted to determine the efficacy of exfoliative cytology in the detection of oral premalignant and malignant lesions. A cytologic diagnosis of the lesions was compared to histopathologic diagnosis of the same lesions. Ninety-one participants were clinically diagnosed as a leukoplakia, 59 were clinically diagnosed as a squamous cell carcinoma, and 40 were clinically diagnosed as normal tissue. The results showed a 77\% specificity, $100 \%$ sensitivity, and $92 \%$ accuracy for those first clinically diagnosed as having leukoplakia. Squamous cell carcinoma displayed a sensitivity of $94 \%$, specificity $100 \%$, and $95 \%$ accuracy 
for those first diagnosed as squamous cell carcinoma. In conclusion, oral exfoliative cytology was determined to be a useful method for detecting oral premalignant and malignant lesions ${ }^{14}$. Oral exfoliative cytology does not replace the need for excisional biopsy as definitive diagnosis.

A study involving 945 patients was performed to evaluate the sensitivity and specificity of the OralCDx brush biopsy. This biopsy uses computer-assisted analysis to determine the cytology of pre-cancerous and cancerous lesions. The study included a double-blind population with patients displaying both suspicious lesions and non-suspicious lesions. An OralCDx brush biopsy was performed on all lesions. Following the OralCDx results, a scalpel biopsy was performed on all lesions having a positive or abnormal result with OralCDx. Results showed that the OralCDx independently detected every histologically confirmed oral dysplasia and carcinoma showing sensitivity $=100 \%$ and the false-negative rate $=0 \%$. The results confirmed that each positive result was a dysplasia or carcinoma. The results showed $100 \%$ specificity for the "positive" results and showed $92.9 \%$ specificity for "atypical" results. The OralCDx brush biopsy found $4.5 \%$ of the "benign-looking" lesions to be dysplasia or carcinoma". This study included no scalpel biopsies on negative lesions.

\section{ORALCDx}

From June 2000 to June 2001, over 40,000 dentists have incorporated OralCDx into their practices $^{16}$. Each OralCDx package includes an instructional video on the performance of the biopsy, 3 OralCDx test kits, a patient education tabletop display and brochures, and a laminated guide to lesions. The test kit includes: a sterile brush biopsy instrument, a bar-coded specimen slide, 2 fixative packets, a slide holder, a bar-coded test requisition form, complete instructions, and a postage-paid return mailing box. Indications for using the OralCDx brush biopsy include the following: 
1. red, white, or mixed lesions

2. chronic ulcerations

3. lesions with unusual surface changes such as a granular appearance

4. evaluation of mucocutaneous disorders

5. follow-up of a persistent lesion despite a benign diagnosis

6. patients with a history of oral or other head and neck cancer and who have evidence of mucosal change ${ }^{17}$.

OralScan Laboratories, Inc. includes a step-by-step guide for performing the biopsy.

Performing the biopsy includes the following steps:

1. Moisten the brush with the patient's saliva.

2. Press the biopsy brush firmly into the lesion, turn the brush 5-10 times until microbleeding occurs.

3. Spread the cellular sample from the biopsy brush to the slide including as much sample from the brush as possible.

4. Pour fixative immediately onto slide and let sit for 15 seconds, then place it in the slide holder and mail the slide to OralScan Laboratories, Inc.

Dentists are facsimiled the results within 3 days after the specimen is received by the laboratory. A screened summary of the representative cellular abnormalities and a pathologist's report is mailed to the dentist for all lesions with positive or abnormal results. These images enable the dentist to explain the abnormal test results to the patient and substantiate the need for further evaluation of the lesion. 


\section{CHAPTER THREE}

\section{METHODS AND MATERIALS}

In November 2001, a 16-question, three page survey (see Appendix C) was mailed through the United States Postal Service to 100 dentists nationally. These dentists were eligible to receive this survey because they had performed a minimum of one OralCDx brush biopsy. In January 2002, a 6-question survey (see Appendix D) was sent to 400 patients who received the OralCDx brush biopsy. These sample populations (dentists and patients) were chosen to survey because of their personal experience utilizing or receiving the OralCDx brush biopsy respectively.

The survey was mailed to 100 dentists, randomly selected from the database of OralScan Laboratories Inc. The names and addresses of the sample populations were sent to the researcher from the Medical Director of OralScan Laboratories Inc. The contact information was destroyed following the completion of the mailing. Surveys did not include any codes to track results because there would be no second mailing. The sample of 400 patients was randomly selected from the database of OralScan Laboratories Inc. In order to maintain complete patient confidentiality, the surveys were sent to the patients directly from the company, returned to, and interpreted by OralScan Laboratories Inc. The company sent the results to the researcher. Results cannot be tracked, therefore, eliminating the possibility of a second mailing.

A cover letter (see Appendix B), a pre-stamped addressed envelope, and a fake \$1 million bill, enclosed as a humorous gesture, were included with each dentist mailing. The cover letter explained that the survey was being conducted in partial fulfillment of the requirements for a Master of Science degree in Dental Hygiene from West Virginia University School of Dentistry. The purpose of this survey was stated, participants were advised that the survey was voluntary, 
and instructions were provided which included the length of time to answer the survey.

Confidentiality was explained and a response deadline of December 20, 2001 was requested.

The 16-item survey for dentists included both multiple choice and completion questions. Questions included demographics, where OralCDx was learned, who performed the biopsy in their office, criteria to perform the biopsy, confidence in the biopsy, number of biopsies performed, percent of biopsies that returned positive or abnormal, and follow-up procedures. The 6-item patient survey included multiple choice and likert scale questions. Content of the questions included high-risk behaviors and habits, understanding of the biopsy procedure, amount of pain felt during the biopsy, fear of the results, and cessation of high-risk behaviors and habits following the biopsy.

Completed surveys were requested to be returned within four weeks from the date they were mailed. At the December deadline, a response rate of $71(71 \%)$ of the dentist surveys and $256(64 \%)$ of the patient surveys was returned. No second mailing was attempted because there was no alternative to track responses received. Data analyses were conducted using the JMP program and included frequencies, cumulative frequencies, percents, analysis of variance, and tests of chi-square. 


\section{RESULTS}

Dentist survey. All (100\%) of the dentists who responded had performed a minimum of one OralCDx brush biopsy, with the majority (73\%) performing 11 or more biopsies. Figure 1 summarizes the number of OralCDx brush biopsies performed by dentists.

Figure 1

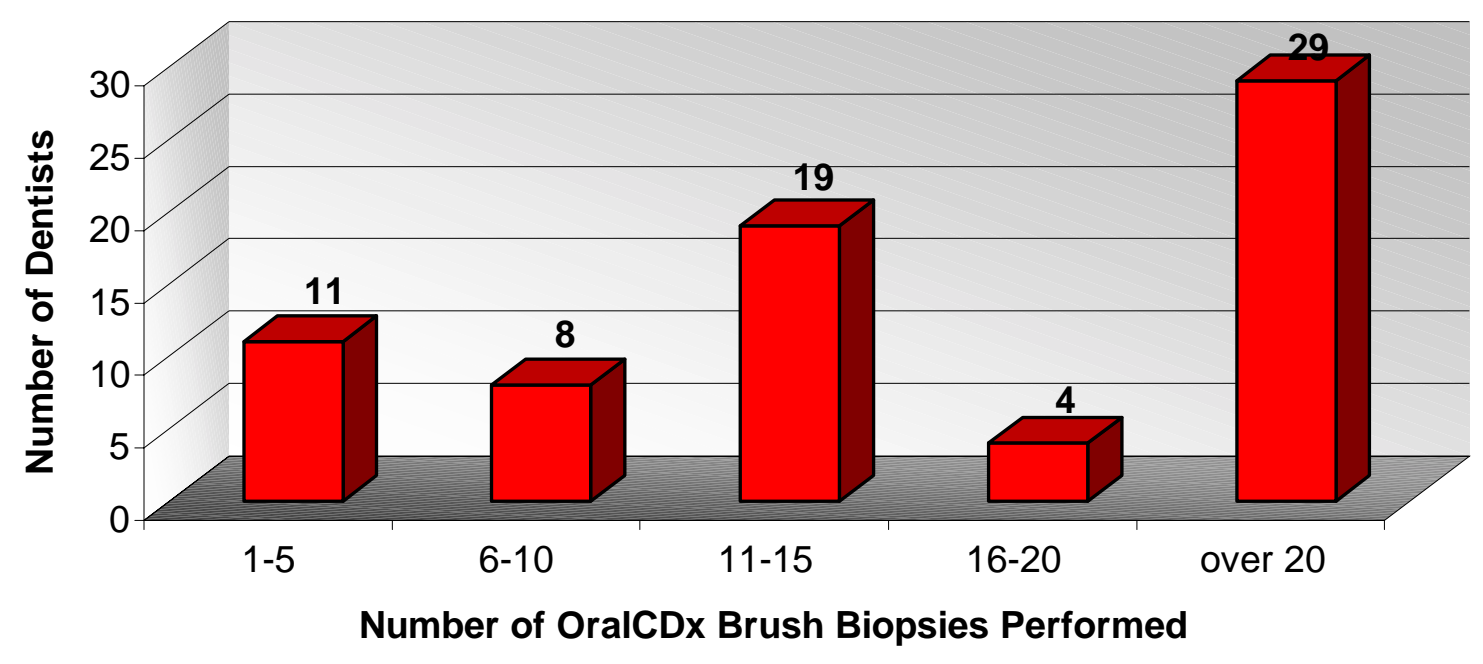

Figure 1. OralCDx Brush Biopsies Performed By Dentists

$(n=71)$ 
Most of the dentists expressed the opinion that all suspicious lesions should be screened with the OralCDx brush biopsy. Figure 2 shows the distribution of dentists who felt that the OralCDx brush biopsy should be used as a screening tool for all suspicious lesions.

Figure 2

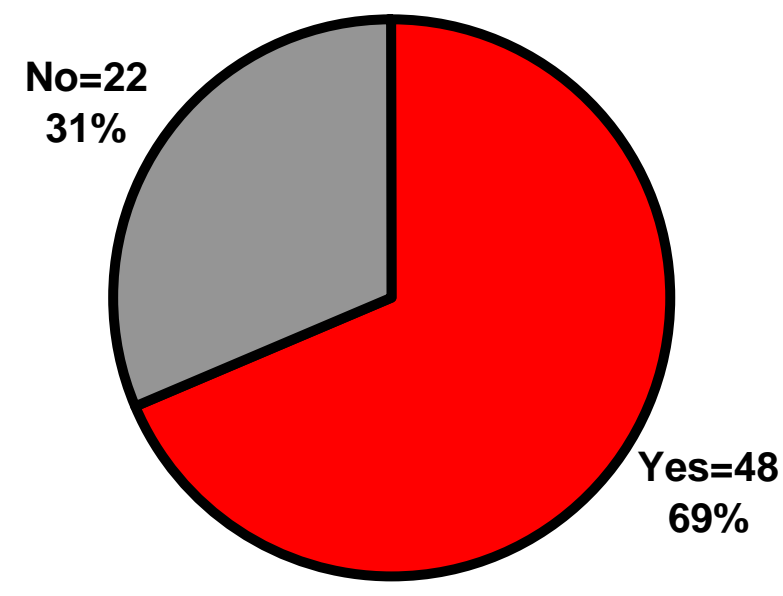

Figure 2. Should All Suspicious Lesions Be Screened with OralCDx? ( $\mathrm{n}=70)$ 
The majority of the dentists had practiced dentistry for 11 or more years, with over half practicing in suburban areas. General dentists followed by Oral and Maxillofacial Surgeons most often included this biopsy procedure in their practices. Figures 3, 4, and 5, respectively, represent the number of years the participants had practiced dentistry, the location of their primary practice, and the type of dentistry they practiced.

Figure 3

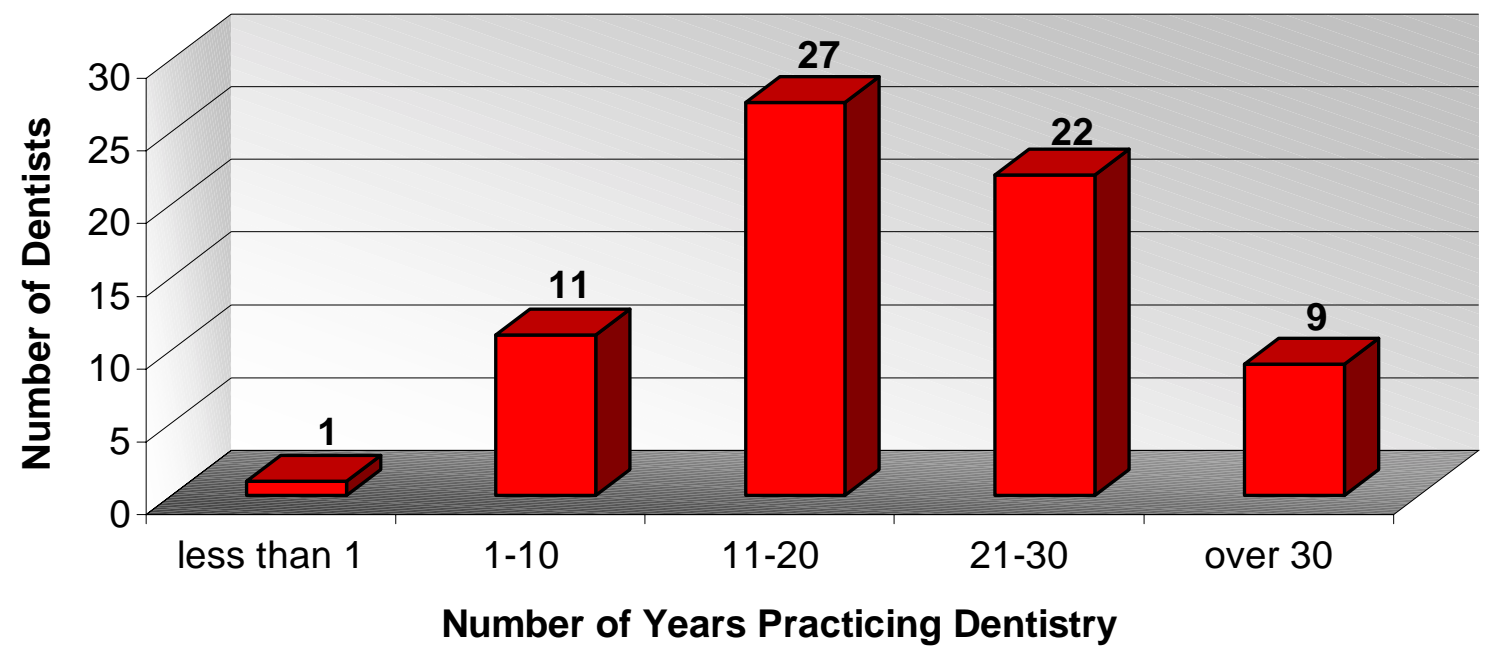

Figure 3. Dentists' Length of Time Practicing Dentistry $(n=70)$ 
Figure 4

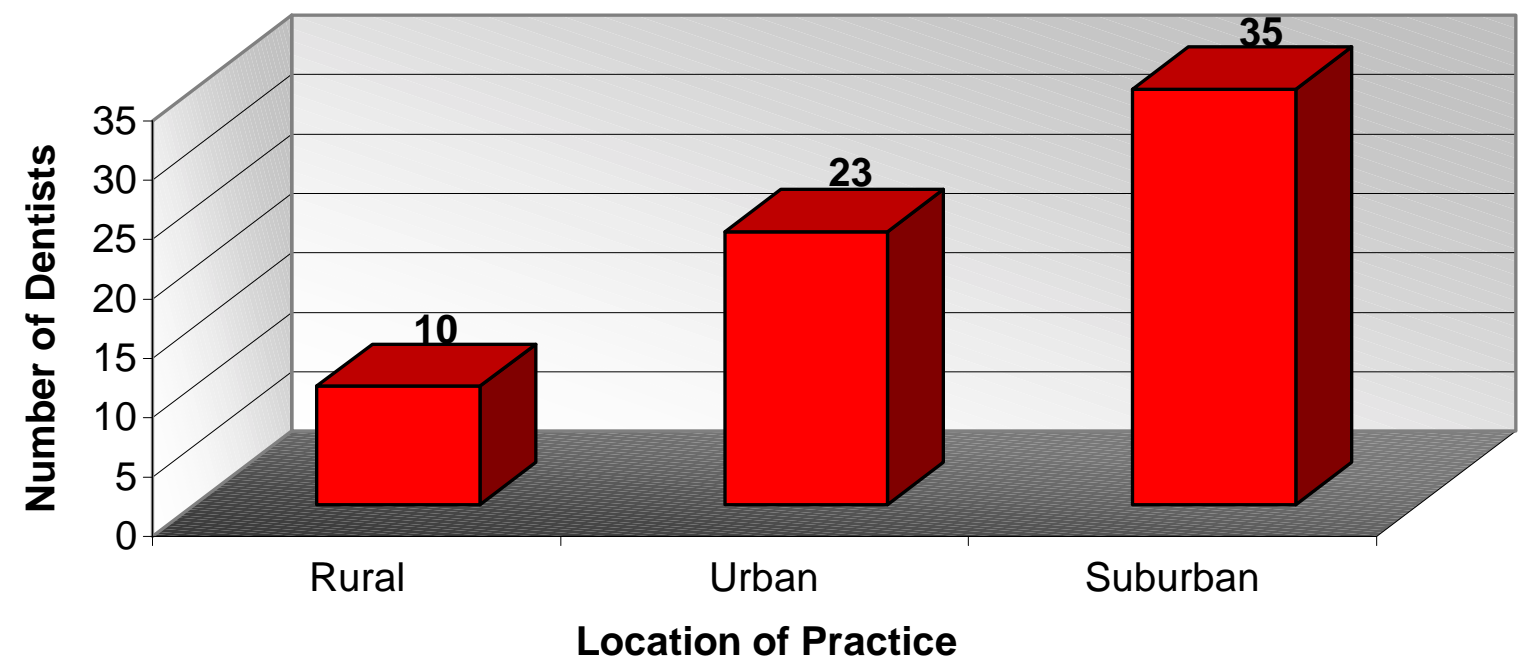

Figure 4. Location of Primary Practice $(n=68)$

Figure 5

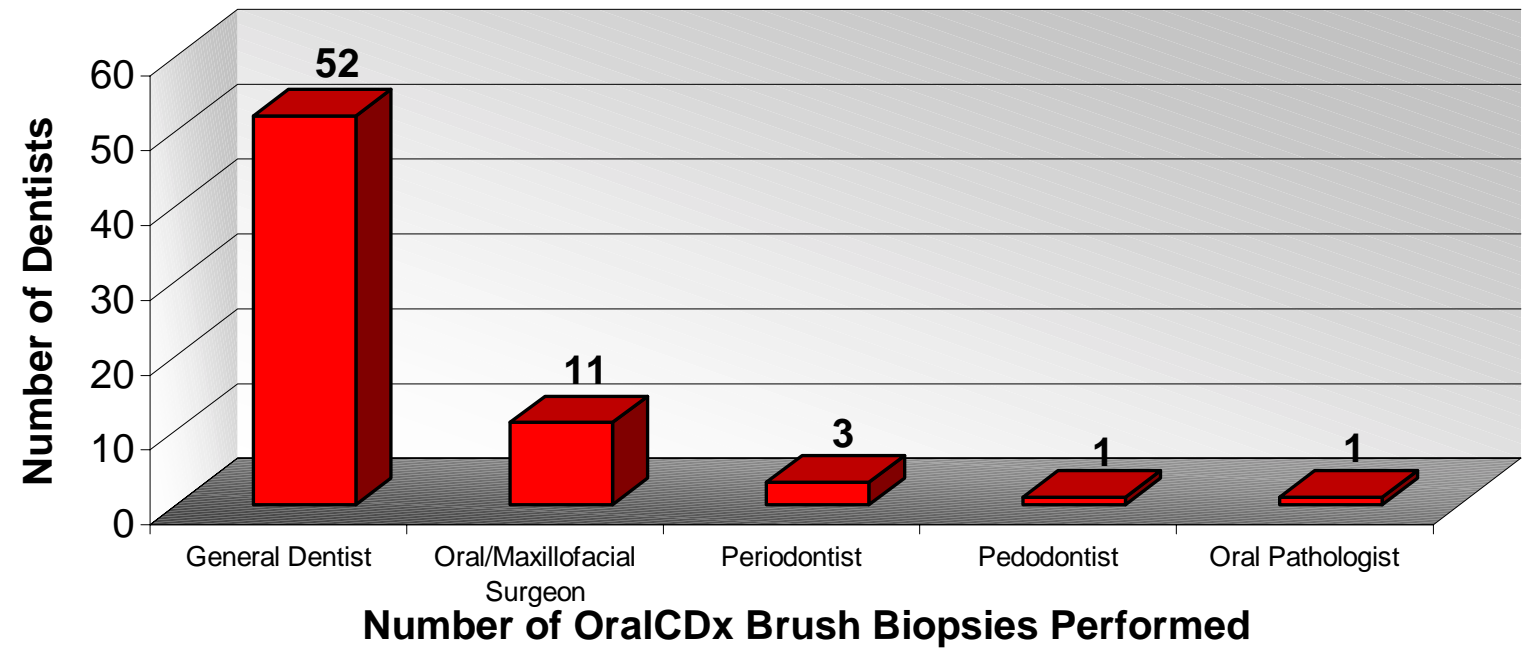

Figure 5. Dentists' Type of Practice $(n=68)$ 
Dentists had performed most of the biopsies, followed by dental hygienists, and dental assistants. Figure 6 shows the proportions of health professionals who performed the OralCDx brush biopsies.

Figure 6

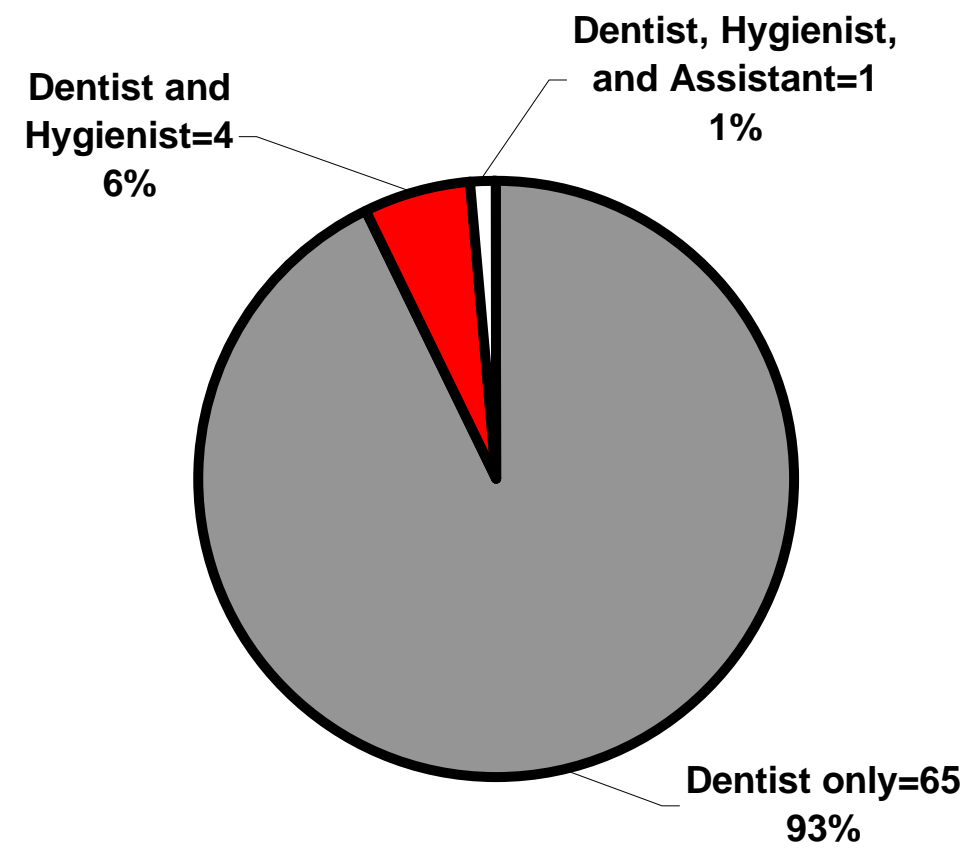

Figure 6. Who Performs the OralCDx Brush Biopsies? $(n=70)$ 
The top four criteria selected for biopsy by respondents included the presence of a leukoplakia or erythroplakia (83\%), the patient with high-risk behaviors and habits $(79 \%)$, the clinical characteristics of the lesion (68\%), and the length of time the oral lesion had been present in the mouth (47\%). Dentists felt that performing a biopsy on a patient may positively motivate that individual to eliminate his or her high-risk behaviors and habits. Figure 7 shows the type of motivation that patients may experience following an OralCDx brush biopsy.

Figure 7

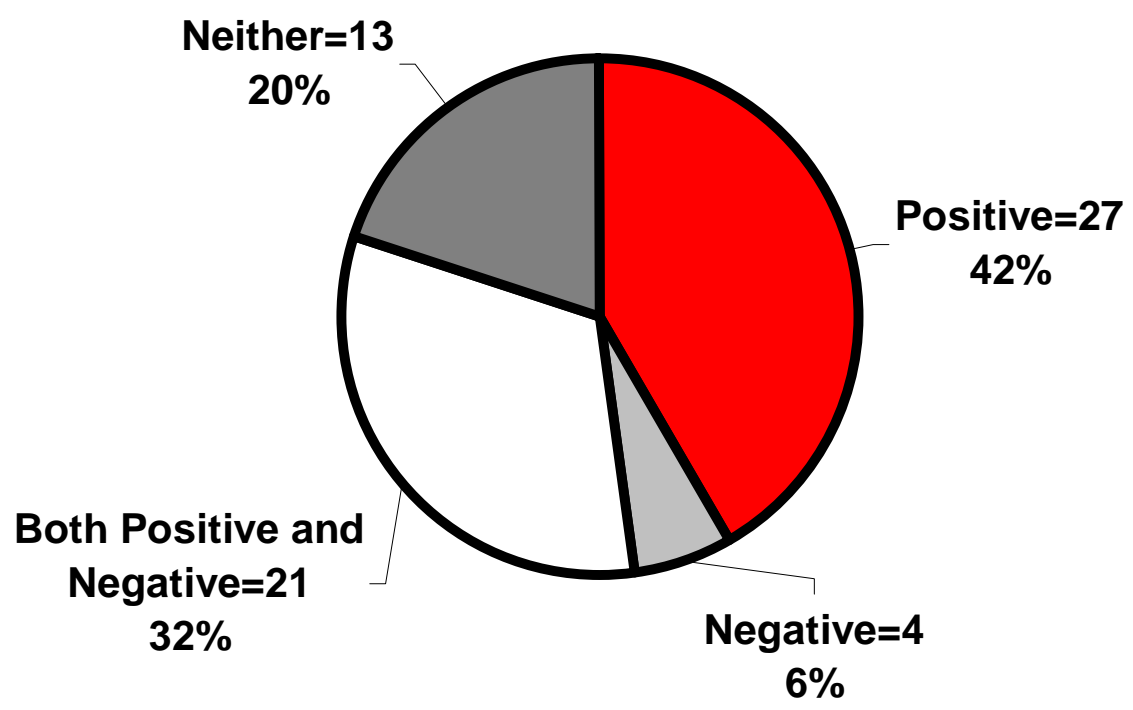

Figure 7. What Type of Motivation Does OralCDx Have on Patients' Behaviors/Habits? ( $n=65)$ 
The majority (74\%) of the participants had high or extremely high confidence in the accuracy of the results of the OralCDx brush biopsy. Figure 8 displays the levels of confidence responding dentists exhibited regarding the accuracy of the OralCDx brush biopsy.

Figure 8

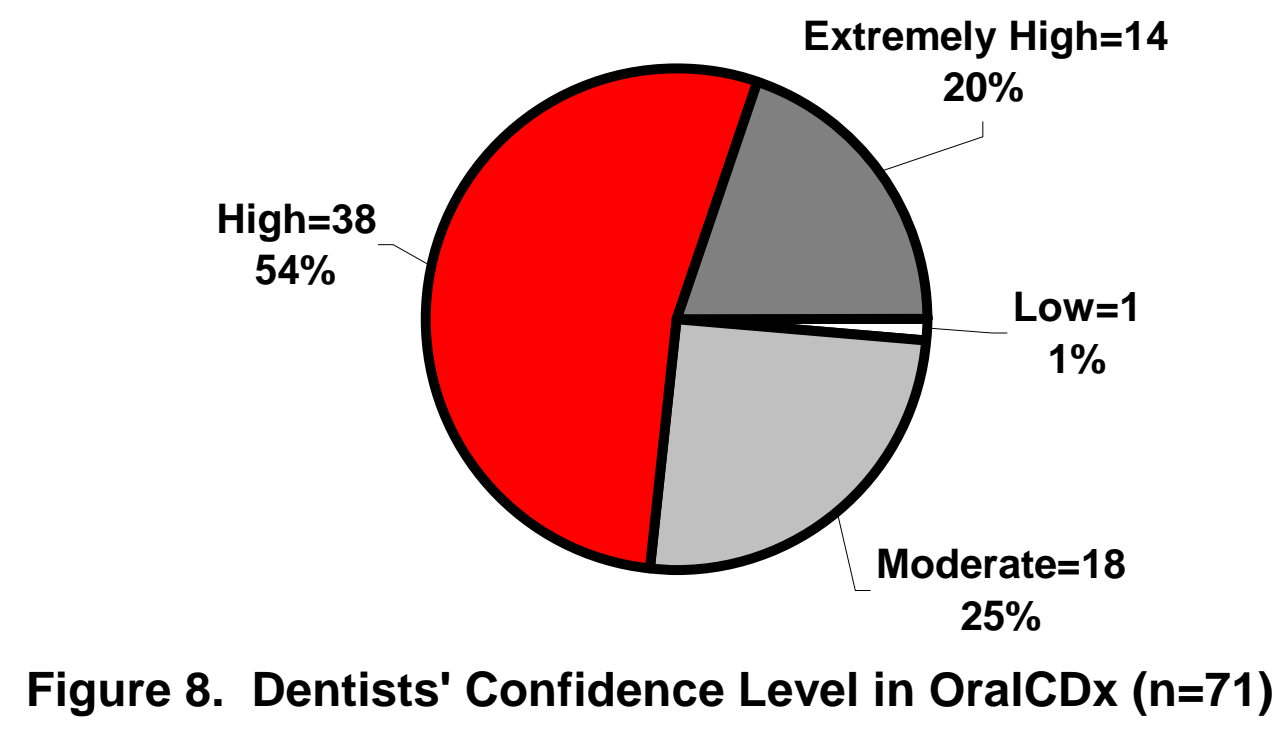


Of the brush biopsies performed by the dentists responding to this survey, less than $20 \%$ exhibited positive or abnormal results. Figure 9 represents the percentage of positive or abnormal biopsy results received by this group.

Figure 9

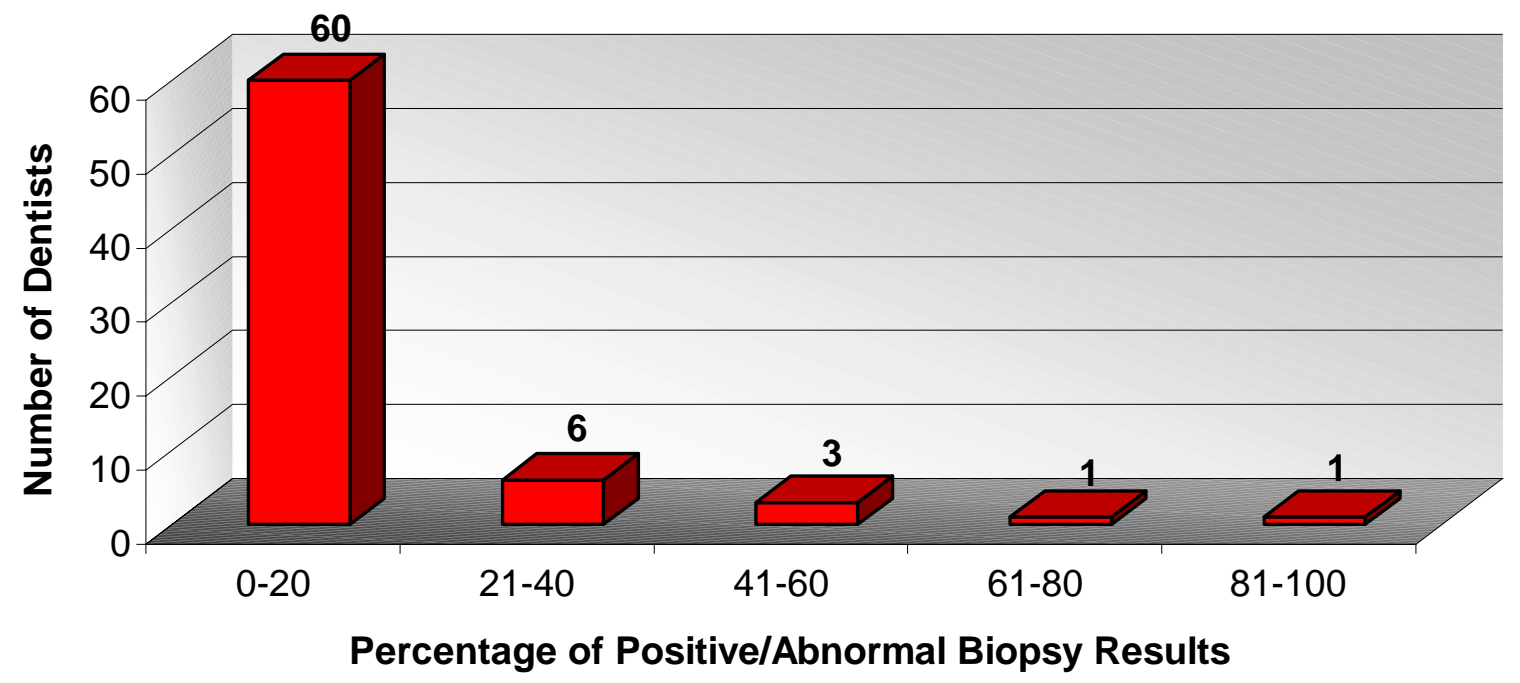

Figure 9. Dentists' Positive/Abnormal Biopsy Results $(n=71)$ 
When positive or abnormal results did occur, the most common follow-up method was to call the patient and schedule and in-office appointment (49\%), followed by a direct referral to a specialist (29\%). Less than half of the dentists surveyed (44\%), performed follow-up procedures that included a direct call to inform the patient of negative lab results following biopsy. Almost all of the dentists reported that they continued to monitor oral lesions that returned with negative results. Figure 10 represents the percentage of dentists that continued to monitor oral lesions that resulted in a negative outcome with the OralCDx brush biopsy.

Figure 10

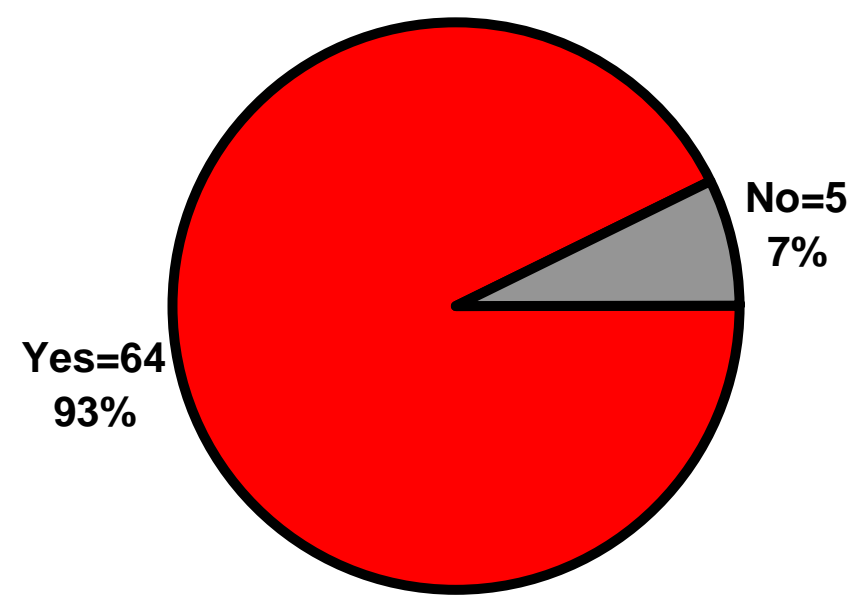

Figure 10. Are Negative Lesions Consistently Monitored Post. biopsy? $(\mathrm{n}=69)$ 
Patient survey. Of the patients surveyed, less than half felt that they had no high-risk behaviors or habits that put them at risk for developing oral cancer(s). Figure 11 summarizes the patients' perceptions of having high-risk behaviors and habits that put them at risk for developing oral cancer(s) prior to receiving the biopsy.

Figure 11

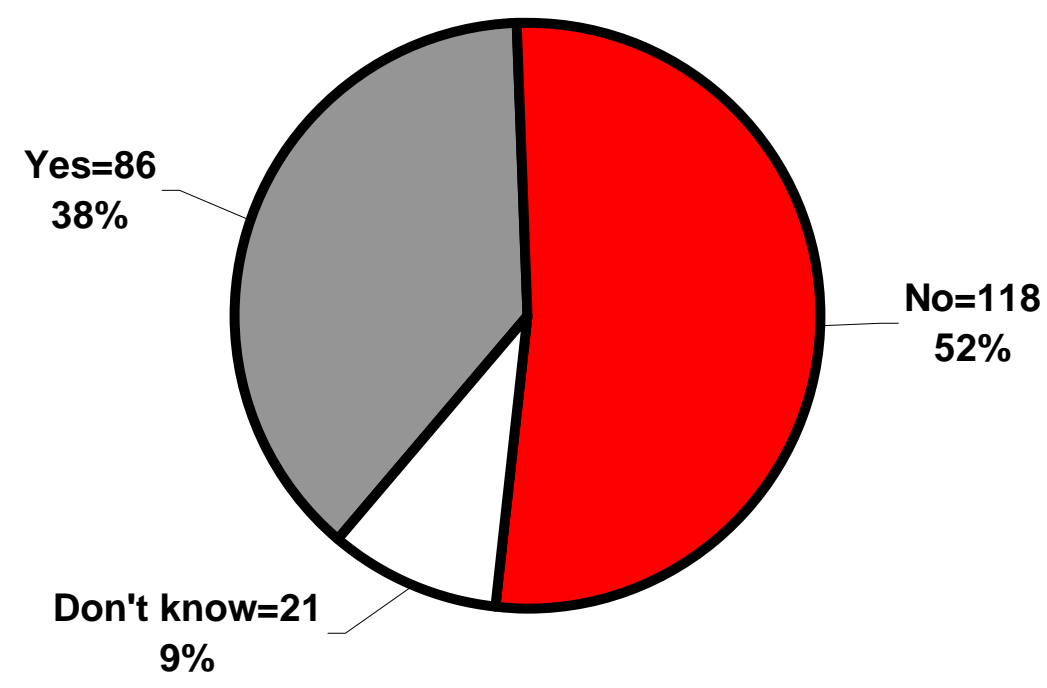

Figure 11. Do Patients' Personal Habits Put Them at Risk for Developing Oral Cancer(s)? $(n=225)$ 
The high-risk behaviors and habits included cigarette smoking (73\%), chewing tobacco (13\%), poor oral hygiene (4\%), not visiting the dentist (1\%), cheek biting (1\%), alcohol use (7\%), and smoking pipe and smoking cigars (1\%). Only (4\%) $n=10$ of the patient respondents reported changing their behavior and habits after undergoing a biopsy procedure. Eight of those individuals stopped smoking (one of which discontinued multiple forms of tobacco) and two ceased their chewing tobacco habit.

Patients had a complete perceived understanding of why they needed to have a biopsy. Figure 12 describes their level of understanding.

Figure 12

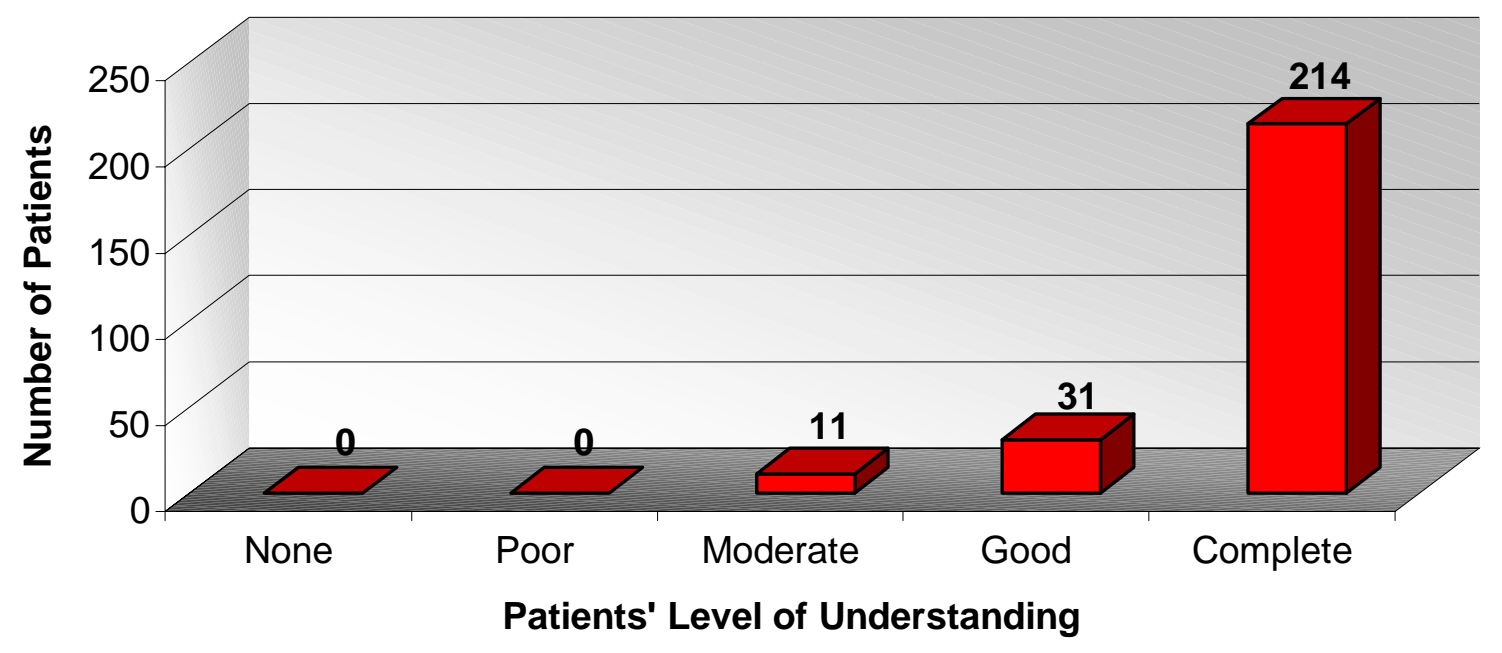

Figure 12. Patients' Understanding of Their Need for an OralCDx Brush Biopsy $(n=256)$ 
The great majority $(88 \%)$ of the patients were not fearful that the results of the biopsy would return positive or abnormal (see Figure 13).

Figure 13

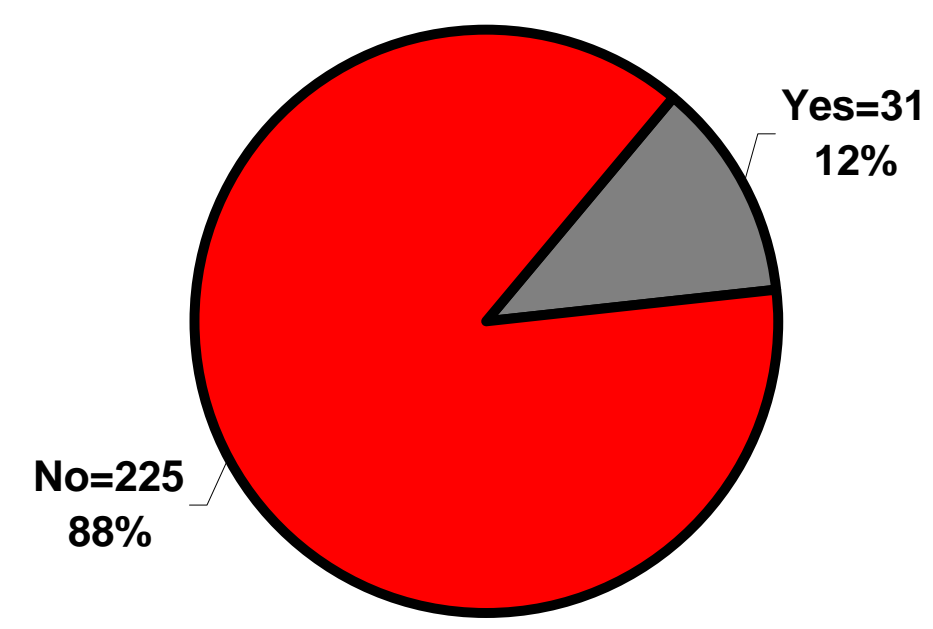

Figure 13. Was the Patient Fearful that Their Biopsy Result would be Positive/Abnormal? $(n=256)$ 
Patients overwhelmingly reported minimal to no pain involvement with the biopsy.

Figure 14 depicts the patients' level of pain involvement with the OralCDx brush biopsy.

Figure 14

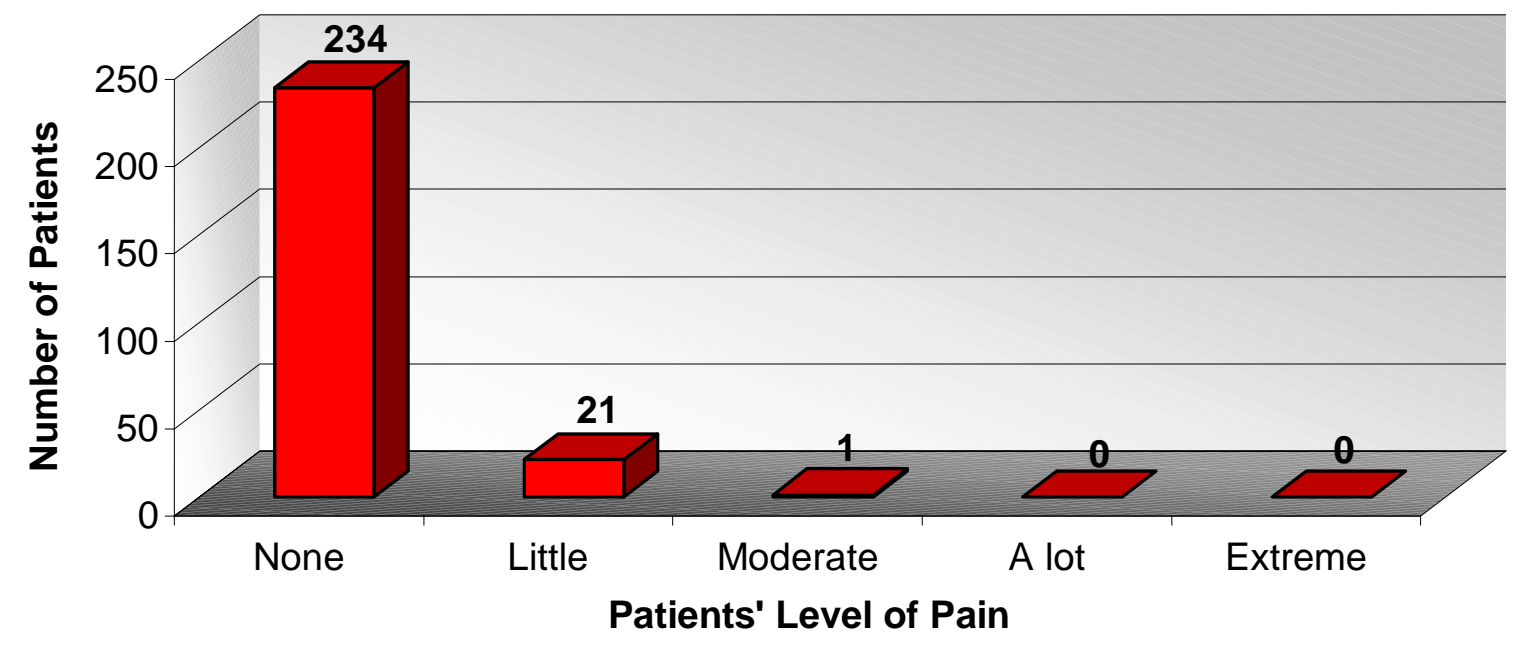

Figure 14. Patients' Pain Involvement with the OralCDx Brush Biopsy $(n=256)$ 
Almost all (99\%) of patients who received the biopsy would recommend the procedure to a friend (see Figure 15).

Figure 15

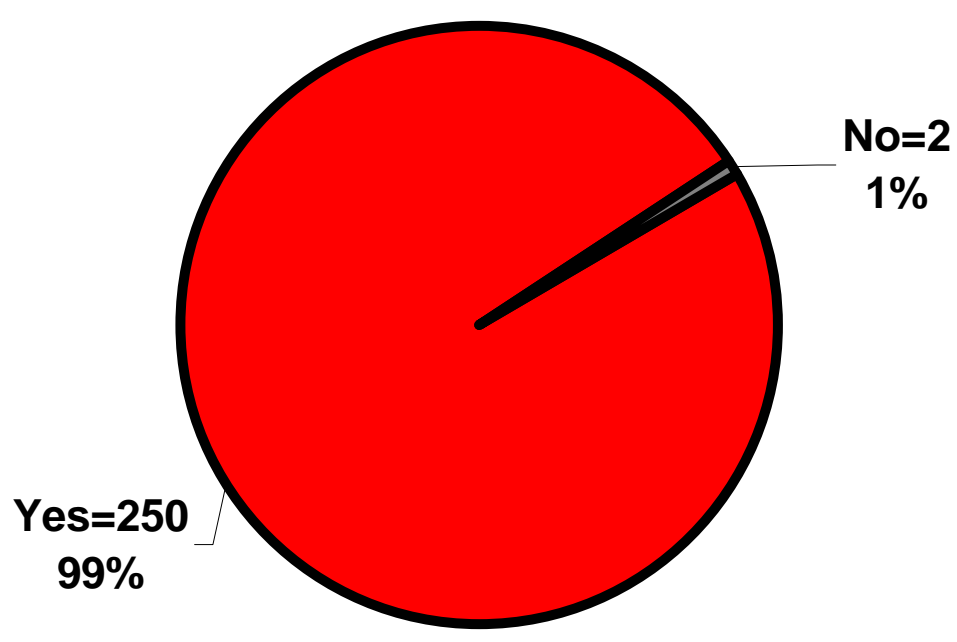

Figure 15. Would Patients Recommend this Procedure to a Friend? $(\mathrm{n}=\mathbf{2 5 2})$ 


\section{DISCUSSION}

The study design did not allow statistical comparison between the patient and dentist survey results. Widespread utilization of the OralCDx brush biopsy does exist and the procedure is perceived to be efficacious due the broad level of acceptance by both the dentist and patient.

Although the criteria for screening with OralCDx is clearly stated by OralScan Laboratories, Inc., the majority of participants (Figure 2) felt that the OralCDx should be used for all suspicious lesions. This data may be misrepresented due to the participants' misunderstanding of the question or the participants not understanding the specific guidelines related to lesion identification for performing the OralCDx brush biopsy.

Participant demographics (Figures 3, 4, and 5) described them as primarily general dentists who are seasoned in their field and are largely located in suburban areas. The investigator speculates that this may be due to the fact that many seasoned professionals may read their professional journals more readily and pursue continuing education. Whereas newer dentists in the field may not have been trained in this technique if it was not discussed in their dental schools. The OralCDx has not been introduced into dental school settings to this point.

While the dentist performs the OralCDx brush biopsy most often (Figure 6), few dentists stated that this task was relegated to dental hygienists in their dental offices. The low number of dental hygienists who were reported to be performing this minimally invasive procedure, may be the result of the varying state practice acts in each state that mandates the expanded functions that a dental hygienist is permitted to perform. The literature that involves early detection of oral cancer is extensive. Since the dental hygienist is the first oral care provider to interview and examine the patient, suspicious oral lesions can be detected early and the minimally invasive OralCDx brush biopsy can subsequently be performed. The investigator suggests that more 
patients may be reached with early oral cancer techniques by allowing dental hygienists to perform the minimally invasive OralCDx brush biopsy.

The majority of dentists, according to the results, selected the top four criteria that indicated biopsy including the existence of a leukoplakia or erythroplakia, the patient with highrisk behaviors or habits, clinical characteristics of the lesion, and the length of time the oral lesion had been present in the mouth. These criteria are included in the oral lesion selection that permits the utilization of the OralCDx brush biopsy by OralScan Laboratories, Inc.

The results showed that less than half of the dentists felt that OralCDx provided positive motivation to patients, and a small subset of patients could be motivated both positively and negatively (Figure 7). The investigator projects that the positive motivation is due to patients proactively being made aware that they are at risk and educating them about steps that could be taken toward changing habits and behaviors, while negative motivation could instill fear or neglect in the patient in which no action would be taken to promote their health.

Although no statistical association was found between the high numbers of OralCDx brush biopsies performed and the high confidence rate dentists had in the procedure, it should be noted that dentists are very confident in the accuracy of the biopsy results (Figure 8). The high confidence that dentists had in the OralCDx brush biopsy exemplifies the need for more dental professionals to be made aware of this procedure in order to detect oral cancers at the earliest possible stage with an accurate, minimally invasive screening tool.

Results of this study showed that almost all (Figure 10) of the dentists continued to monitor oral lesions that were found to have a negative result with OralCDx. This is commendable due to the fact that at this point, no literature exists concerning the results of a 
scalpel biopsy in conjunction with the OralCDx brush biopsy to identify the specificity and sensitivity for negative oral lesions with the OralCDx brush biopsy.

Although, over $25 \%$ of oral cancer victims have not used tobacco and had no other known high risk factors ${ }^{3}$, results revealed that over half of the patients surveyed perceived themselves as having no high-risk habits or behaviors that put them at risk for developing oral cancers (Figure 11). This could have resulted due to the fact that the investigator did not have complete control of the patient selection, or it could have resulted from the patients' perception of what high-risk factors include.

Results showed that almost all of the patients surveyed felt that they had complete understanding of the procedure and why they needed a biopsy (Figure 12). Although no literature exists to support this claim, the investigator speculates that this finding could be attributed to the overall understanding of the procedure by the dentist and their ability to explain it to their patient, the faith the patient had in the doctor's understanding of the procedure, the patient not understanding the survey question, or because of the patient not wanting to admit a lack of understanding.

The majority of the patients surveyed were not fearful of the results of their biopsy (Figure 13). This could be attributed to the patients' complete understanding of their need for the biopsy and the procedure involved. Having a complete understanding about the procedure they will experience and what the biopsy screens for may put the patient at ease and reduce their fear of a positive OralCDx brush biopsy.

Perhaps the highest level of acceptance was the recommendation of the procedure to a friend or family member. Having a complete understanding of the procedure, minimal to no pain 
involvement, no fear of the results, and having no perceived high-risk behaviors or habits could all contribute to the patients' support of the OralCDx brush biopsy. 


\section{SUMMARY AND CONCLUSION}

Based on the data collected, literature reviewed, and the investigator's experience, the OralCDx brush biopsy 1) is an accepted screening tool by both dental professionals and patients, 2) is used with frequency by those professionals who had performed the procedure it at least once, 3 ) is minimally invasive in nature, 4) is relatively easy to use, 5) is a time efficient tool, 6) is accurate in providing results according to published literature, and 7) appears to be a technique that could be performed by dental hygienists. Therefore, education about the utilization and efficacy should be shared among dental professionals and their patients in order to promote health and longevity. From this study, the following may be concluded:

1. Both dentist and patient have confidence in the efficacy of the OralCDx brush biopsy.

2. Patients understand why the OralCDx brush biopsy is necessary in the early detection of suspicious oral lesions.

3. Patients did not experience pain involvement associated with the OralCDx brush biopsy.

4. Dentists and patients would recommend the OralCDx brush biopsy to their family or a friend.

5. The OralCDx brush biopsy is utilized by a variety of dental professionals.

6. The OralCDx brush biopsy is simple to use. 


\section{RECOMMENDATIONS}

1. In order to determine sensitivity and specificity of OralCDx, a study should be designed to screen for both positive and negative lesions with the OralCDx brush biopsy followed by incisional biopsy.

2. The OralCDx brush biopsy should be incorporated into general and oral pathology courses to enable health science students to gain knowledge of the procedure, use, and indication for OralCDx as a minimally invasive screening for suspicious oral lesions.

3. A subsequent study should be conducted that involves dentist and patient perceptions of OralCDx efficacy and utilization after the procedure is more established by overall dental professionals.

4. A survey should be developed to compare a stratified random sample of dentists who have performed the OralCDx brush biopsy, with dentists who have not performed the OralCDx, in order to gain understanding of perceived efficacy and utilization of the procedure by both groups.

5. A study should be conducted nationally to determine the professional efficacy and utilization by dental hygienists that have performed a minimum of one OralCDx brush biopsy.

6. For this study, the researcher recommends a larger randomly stratified dentist and patient sample size and a list of high-risk behaviors to be included on the patient survey to enable the patient to select each high-risk behavior that they have in order to minimize subjectivity.

7. The name OralCDx brush biopsy may need to be re-evaluated in order to allow dental hygienists to perform this procedure according to the guidelines associated with insurance and legal issues. 


\section{REFERENCES}

1. Clark, A. Oral Cancer Prevention and Early Detection. Nursing Standard 1999; 13:431.

2. Preventing and Controlling Oral and Pharyngeal Cancer: Recommendations from a National Strategic Planning Conference. MMWR: Morbidity and Mortality Weekly Report 47(RR-14), 1-12. 1998. 4-17-2002.

Ref Type: Electronic Citation

3. Oral Cancer Facts. http://www.oralcdx.com/oralcancer facts.html , 1-2. 2001. OralScan Laboratories, Inc. 10-15-2001.

Ref Type: Electronic Citation

4. ACS::What is Oral Cavity and Oropharyngeal Cancer? http://www.cancer.org/eprise, 2-3. 2002. 10-29-2001.

Ref Type: Electronic Citation

5. Neville, B., Damm, D., Allen, C., Bouquot, J. Oral and Maxillofacial Pathology, 2nd ed. Philadelphia: WB Saunders; 2002. p. 315-87.

6. Ship, JA. et al. Evaluation and Management of Oral Cancer. Home Health Care Consultant 1999;6:2-5, 9-12.

7. Dushanka, V. et al. Cancers of the Oral Cavity and Pharnyx: A Statistics Review Monograph, 1973-1987. Kleinman, Dushanka V. et al. 1991. Atlanta, GA: U.S. DHHS, PHS, CDC; Bethesda, MD: NIH. DHHS Publication; no (PHS) 91-50212.

Ref Type: Serial (Book,Monograph)

8. Boyle, P. et al. Oral Cancer: Necessity for Prevention Strategies. Lancet 1993; 342:1129.

9. Tobacco Use--United States, 1900-1999. MMWR: Morbidity and Mortality Weekly Report 1999; 48:986-93.

10. Second Cancers Following Oral and Pharyngeal Cancers: Role of Tobacco and Alcohol. Journal of the National Cancer Institute 1994;86:131-37.

11. Mucci, LA., Brooks, DR. Lower Use of Dental Services Among Long Term Cigarette Smokers. Journal of Epidemiology and Community Health 2001;55:389-93. 
12. Bouquot, JE., Meckstroth, RL. Oral Cancer in a Tobacco-chewing US Population--No Apparent Increased Incidences or Mortality. Oral Surgery, Oral Medicine, Oral Pathology, Oral Radiology, and Endodontics 1998;86:697-706.

13. Rankin, KV,. et al. Oral Cancer Education In Dental Schools: Survey of Texas Dental Students. Journal of Cancer Education 1996;11:80-83.

14. Ramesh, T. et al. Exfoliative Cytology in Screening for Malignant and Premalignant Lesions in the Buccal Mucosa. The Ceylon Medical Journal 1998;43:206-09.

15. Sciubba J. Improving Detection of Precancerous and Cancerous Oral Lesions: Computerassisted Analysis of the Oral Brush Biopsy. Journal of the American Dental Association 1999;130:1445-57.

16. Good News About Early Detection. http.//www.oralscan.com/good news.html, 1-3. 2001. 112-2001.

Ref Type: Electronic Citation

17. For Dentists. http://www.oralscan.com/dentists.dentists.htm, 1-3. 2001. 11-2-2001. Ref Type: Electronic Citation 


\section{APPENDIX A}

INSTITUTIONAL REVIEW BOARD APPROVAL FOR THE PROTECTION OF HUMAN SUBJECTS 


\section{APPENDIX A}

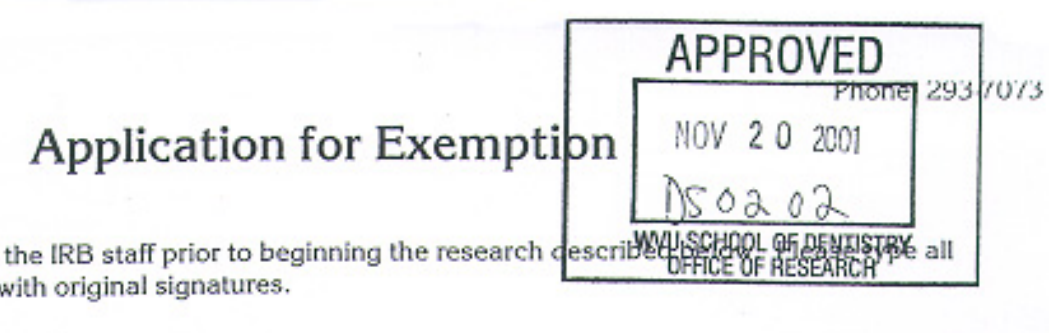

You must receive approval from the IRB staff prior to begi
responses and submit this form with original signatures.

1. Title of study: Qperater Efficacy and Utilization of the QralCDx Brush Biopsy.

2. Investigators (list all investigators, principal investigator first; attach additional sheets if necessary):

\begin{tabular}{|l|l|l|l|l|}
\hline Name & \multicolumn{1}{c}{ Signature } & \multicolumn{1}{c|}{ Address } & \multicolumn{1}{c|}{ Tel. No. } \\
\hline Courtney Huffman & & Dental Hygiene & $1188 \mathrm{HSN}$ & $3-3922$ \\
\hline Christina DeBiase & gental Hygiene & $1188 \mathrm{HSN}$ & $3-3922$ \\
\hline
\end{tabular}

3. Estimated period of human subject involvement: Starting date 11-20-2001

Ending Date 5-30-2002

4. Reason for conducting research $\quad \square$ Professional $\quad \square$ Dissertation

$\nabla$ Thesis

5. Source of funding (if applicable) WVU SOD Research Corporation

$$
\text { specify }
$$

6. Number of projected subjects $100 \quad$ Number of projected records or data files

7. This research involves (check all that apply — see attached "Exempt Research" page):

- a. collection or study of existing data, documents, records or specimens, recorded without identifiers

— b. normal educational practices conducted in established or commonly accepted educational settings

_ c. educational tests (cognitive, diagnostic, aptitude, achievement)

- d. observation of public behavior

$\checkmark$ e. surveys, interviews or hand-outs for subjects over 18 (use age ranges, not actual age, for demographic information)= $\square$ mail $\square$ telephone $\square$ person-to-person

_f. any possibility of identifying a subject (discuss in cover letter)

- g. the possibility that the subject's responses or conduct (if they became public) may place the subject at risk of criminal or civil liability or be damaging to the subject's financial standing or employability

_ h. sensitive aspects of personal behavior (for example: illegal conduct, drug use, sexual behavior or use of alcohol)

_ i. investigator's participation in activities being observed

- j. only surveys or interviews of elected or appointed public officials or candidates for public office

_ k. audiotaping

- I. children under age 18 (see Chapter II of the Guidelines) Note: Interviews and surveys with children are never exempt.

_ m. food tasting and evaluation

n. research and demonstration projects

8. Goal of research

The goal of this research is to identify efficacy and utilization of the GralCDx Brush Biopsy as a necessary and heneficial screening tool prior to incisional biopsy of suspicious aral lesions identified by diental professionals 
9. Explanation of procedures involved in research

Medical Ditector, Dr. Drore Fisen, will randomly select 100 dentists that have nerformed the bionsy nationwide from OralScan Laboratories. A 16-item survey will be sent out to those 100 dentists selected to assess utilization and efficacy of the biopsy.

10. Explanation of known risks to hurnan subjects

Sunveys will include directions, a cover letter including voluntary particination, names are NOT to be included on the surveys, and that surveys will be keot confidential. There will be no second mailings to track results.

11. Explanation of how records will be kept

All documents will be kept in the possession of the investigator and destroyed upon complation of the study.

A cover letter addressed to respondents must accompany any survey or questionnaire. The cover letter must be on your WVI departmental fetterhead and must include the following:

1. a statement that the project is research being conducted in partial fulfillment of the requirements for a course, master's thesis, dissertation, etc.

2. purpose of study

3. a statement that subjects' responses will be kept anonymous or confidential (explain extent of confidentiality if subjects' names are requested)

4. If audiotaping, a statement that subject is being audiotaped (explain how tapes will be stored or disposed of during and after the study)

5. a statement that subjects do not have to answer every question

6. a statement that subject's class standing, grades, or job status (or status on an athletic team, if applicable) will not be affected by refusal to participate or by withdrawal from the study

7. a statement that participation is voluntary

Attachments:

$\checkmark$ questionnaire/survey to be used

_ telephone text (including introductory remarks as in a cover letter-see above)

$\underline{v}$ cover letter

— permission from external institution, on their letterhead (if applicable)

I have reviewed the above information and recommend this study for exemption.

Dean or Difector

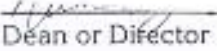

Department Chair
Faculty Advisor 
$\mathrm{X}$ _questionnaire/survey to be used

_telephone text (including introductory remarks as in a cover Ietter-ee above)

$\mathrm{X}$ cover letter

$\mathrm{X}$ permission from extemal institution, on their letterhead (if applicable)

1 have reviewed the above information and recommend this study for exemption. 


\section{APPENDIX B}

COVER LETTER - DENTISTS 


\section{APPENDIX B}

\section{WestVirginiaUniversity}

SCHOOL OF DENTISTRY

November 19,2001

\section{APPROVED \\ NOV 202001 \\ $\triangle 502.02$ \\ WNU SCHOOL OF DENTISTRY OFFCE OF RESEARCH}

Dear Doctor,

As you know, oral cancer has the best long-term prognosis when diagnosed early. The oral brush biopsy is a screening tool designed to detect oral lesions at an early stage. You have been anonymously selected from the OralCDx databasc, of OralScan Laboratories, to participate in this research study because you have performed this procedure in the past. The purpose of this study is to identify your experiences regarding the efficacy and utilization of the oral brush biopsy technique. This study is being performed as partial fulfillment for my master's thesis in dental hygiene at West Virginia University. Your time is very valuable and therefore, this survey should take no more than ten minutes to complete.

Your participation is voluntary. Neither you nor my grade will be affected by your refusal to participate. You are not required to respond to every question although the completeness of your responses will be helpful. All responses will be kept as confidential as legally possible.

I have enclosed a self-addressed, stamped envelope for your convenience upon completion of this survey. Please return your responses by December 20, 2001. Also, as an incentive, I have enclosed a $\$ 1,000,000$ bill as a humorous gesture for your time and contribution to my study.

Thank you for your time and participation.

Sincerely,

Courtney N. Huffmad RDH

Master's Candidate

- manyo.

Richard L. Meckstroth DDS

Chairman Department of Dental Practice and Rural Health

\author{
Department of Dental Practice and Rural Health \\ G110 Health Sciences North \\ PO Box 9415 \\ Norgantown, WW 26506.9415
}

41

Equal Opportunityatlinmative Action instiotion 


\section{APPENDIX C}

SURVEYS - DENTISTS 


\section{APPENDIX C}

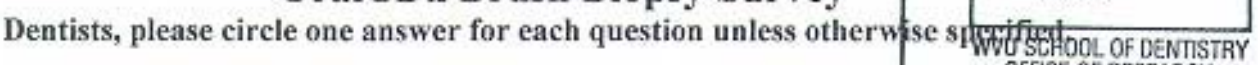

1. How long have you been practicing dentistry?
A. less than a year
B. $1-10$ years
C. $11-20$ years
D. $21-30$ years
E. over 30 years

2. Which of the following best describes the location of your practice:
A. Rura
B. Urban
C. Suburb

3. Which of the following best describes your primary practice:
A. General Dentistry
B. Oral/Maxillofacial Surgery
C. Periodontics
D. Pediatrics
E. Prosthodontics
F. Orthodontics
G. Endodontics
H. Oral Pathology
I. Oral Medicine

4. Where did you learn about the brush biopsy technique?
A. C.E. Course
B. Journal article
C. Professional Colleague
D. Advertisement
E. Other, please explain

5. Who performs the brush biopsies in your office? (Circle all that apply)
A. Dentist
B. Dental Hygienist
C. Dental Assistant
D. Other, please list 
6. Circle up to four criteria that you use to determine when you will perform a brush biopsy:
A. Small lesion size
B. Clinical characteristics of lesion (appearing "harmless")
C. High risk patient behaviors/habits
D. Existence of leukoplakias or erythroplakias
E. Age of patient
F. Pain involvement
G. Length of time lesion is present
H. History of immunocompromising condition
I. Patient refuses scalpel biopsy
J. Other, please list

7. What degree of confidence do you have that the results of the brush biopsy procedure are accurate?
A. Low
B. Moderate
C. High
D. Extremely High

8. Approximately how many brush biopsies have you performed?
A. $1-5$
B. $6-10$
C. $11-15$
D. $16-20$
E. over 20

9. Based on the number of brush biopsies you reported performing in question 8 , what percentage of those biopsies had positive/abnormal results?
A. $0-20 \%$
B. $21-40 \%$
C. $41-60 \%$
D. $61-80 \%$
E. $81-100 \%$
F. Unknown

10. When a biopsy result is positive/abnormal, what is your initial policy for follow-up?
A. Call patient directly to schedule an in-office consultation
B. Send a letter to the patient informing them of the results
C. Make a direct referral to a specialist
D. Other, please explain 
11. When a biopsy result is negative, what is your initial policy for follow-up?

A. Call patient directly to schedule an in-office consultation

B. Send a letter to the patient informing them of the results

C. Make a direct referral to a specialist

D. Other, please explain

12. Who do you feel that the oral brush biopsy benefits: (Circle all that apply)
A. Patient
B. Doctor
C. Both the patient and the doctor
D. Neither the patient nor the doctor

13. Do you feel that the brush biopsy is a valuable screening tool for all suspicious lesions?
A. YES
B. NO

14. The brush biopsy can motivate patients to eliminate high-risk behaviors in various ways. What type(s) of motivation have your patients experienced since having this biopsy?
A. Positive motivation only (i.e. increase patient's awareness)
B. Negative motivation only (i.e. increase patient's fear of cancer)
C. Both positive and negative motivation
D. Unchanged

15. Do you continue to monitor persistent oral lesions that have had negative results with the brush biopsy?
A. YES
B. NO

16. Would you perform this procedure on a family member utilizing the criteria you selected in question 6 ?
A. YES
B. NO 


\section{APPENDIX D}

SURVEY - PATIENT 


\section{APPENDIX D}

\section{OralCDx Brush Biopsy Survey}

Please circle only one answer for each question unless otherwise specified.

1. Prior to receiving the brush biopsy, did you have any personal habits and/or behaviors that put you at risk for developing oral cancer(s)?
A. Yes
B. No

If you answered NO to question \#1 go directly to question \#3. If you answered YES to question \#1, please circle all the habits and/or behaviors you had prior to receiving the brush biopsy:
A. cigarette smoking
B. cigar smoking
C. tobacco chewing/snuff
D. pipe smoking
E. excessive alcohol drinking
F. Other, please list

2. How well did you understand why you needed the brush biopsy procedure?

\begin{tabular}{|c|c|c|c|c|}
\hline 1 & 2 & 3 & 4 & 5 \\
\hline $\begin{array}{l}\text { No } \\
\text { Inderstanding }\end{array}$ & $\begin{array}{c}\text { Poor } \\
\text { Understanding }\end{array}$ & $\begin{array}{c}\text { Moderate } \\
\text { Understanding }\end{array}$ & $\begin{array}{l}\text { Good } \\
\text { Understanding }\end{array}$ & $\begin{array}{c}\text { Complete } \\
\text { Understanding }\end{array}$ \\
\hline
\end{tabular}

3. Circle the amount of pain you felt during the brush biopsy?

$\begin{array}{ccccc}1 & 2 & 3 & 4 & 5 \\ \text { No pain } & \text { A little pain } & \text { Moderate pain } & \text { A lot of pain } & \text { Extreme pain }\end{array}$

4. Were you fearful that the results of your brush biopsy would return positive/abnormal?
A. Yes
B. No

5. Have you changed any of your habits and/or behaviors now that you have had the biopsy?
A. Yes
B. No 
If you answered No to question \#5 go directly to question $\# 6$. If you answered Yes to question \#5, please circle all of the behaviors and/or habits you have changed since having the brush biopsy

procedure:
A. decreased or stopped smoking cigarettes
B. decreased or stopped smoking cigars
C. decreased or stopped using chewing tobacco/snuff
D. decreased or stopped smoking pipes
E. decreased or stopped drinking alcohol
F. Other, please list

6. Would you recommend the brush biopsy procedure to a friend?
A. Yes
B. No

Please feel free to add any additional comments or suggestions you may have concerning this survey or any additional experiences you had with the brush biopsy procedure:

Thank you for you time and consideration! 


\section{VITA}

\section{Courtney Niccole Huffman}

\section{PERSONAL DATA}

Parents: Thomas and Deborah Keith

Birthplace: Waynesburg, PA

Birthdate: $\quad$ September 12, 1977

\section{PROFESSIONAL EDUCATION}

West Virginia University, Morgantown, WV 1996-2000

Bachelor of Science in Dental Hygiene

West Virginia University, Morgantown, WV 2000-2002

Master of Science in Dental Hygiene

\section{PROFESSIONAL EXPERIENCE}

Dr. Leslie Midla, Beallsville, PA

West Virginia University

Oral and Maxillofacial Clinic

West Virginia University

Gross Anatomy Laboratory Graduate Instructor

West Liberty State College

Dental Hygiene Clinical Graduate Instructor

West Virginia University

Dental Hygiene Clinical Graduate Instructor

West Virginia University

Oral and Dental Anatomy Classroom and Laboratory Graduate Instructor 